\title{
Fast and Accurate Modeling of Dual-Polarized Reflectarray Unit Cells Using Support Vector Machines
}

\author{
Daniel R. Prado, Jesús A. López-Fernández, Guillermo Barquero, Manuel Arrebola, Senior Member, IEEE, \\ and Fernando Las-Heras, Senior Member, IEEE
}

\begin{abstract}
This paper describes the characterization of reflectarray unit cells using Support Vector Machines (SVMs) to obtain fast and accurately the full matrix of reflection coefficients, which is used for an analysis of dual-polarized reflectarrays, demonstrating the performance of the model. First, a surrogate model of the reflectarray unit cell is obtained using SVMs. To this end, a set of random samples of the reflection coefficient matrix with a full-wave Method of Moments based on Local Periodicity (MoM-LP) is used to train the SVMs. To efficiently obtain the surrogate model, a novel strategy to accelerate the training process is presented, remarkably reducing computing time. Next, the model is tested against a different set of samples, obtaining an excellent agreement between the SVM model and MoM-LP simulations for all reflection coefficients, including the crosscoefficients. The surrogate model is then used for an efficient analysis of three reflectarrays with pencil beam for point-topoint communications, isoflux pattern for global Earth coverage, and a shaped-beam for Local Multipoint Distribution Service application, showing excellent agreement in both copolar and crosspolar patterns between the SVM and MoM-LP simulations. Finally, the analysis is accelerated by a factor larger than three orders of magnitude using SVMs instead of MoM-LP.
\end{abstract}

Index Terms-Support Vector Machine (SVM), unit cell, efficient analysis, reflectarray, shaped beam antenna, point-to-point communications, Local Multipoint Distribution Service (LMDS), isoflux pattern

\section{INTRODUCTION}

$\mathbf{R}$ EFLECTARRAY antennas have been widely studied in the last three decades since the popularization of the microstrip technology [1]. This kind of antenna unifies the worlds of the parabolic reflectors and phased-arrays, overcoming some of their drawbacks and resulting in a low mass, low volume and low profile antenna with great capabilities of beamforming while keeping low losses [1]. The main drawback of reflectarray antennas is their inherent narrow bandwidth, although it can be overcome by employing broadband elements

This work was supported in part by the European Space Agency (ESA) under contract ESTEC/AO/1-7064/12/NL/MH; by the Ministerio de Economía y Competitividad under project TEC2014-54005-P (MIRIIEM); by the Gobierno del Principado de Asturias / FEDER under project GRUPIN14-114.

D. R. Prado, J. A. López-Fernández, G. Barquero, M. Arrebola and F. LasHeras are with the Department of Electrical Engineering, Group of Signal Theory and Communications Universidad de Oviedo, Gijón 33203, Spain (e-mail: drprado@tsc.uniovi.es; jelofer@tsc.uniovi.es; arrebola@tsc.uniovi.es; flasheras@tsc.uniovi.es).

Color versions of one or more of the figures in this paper are available online at http://ieeexplore.ieee.org.

Digital Object Identifier XX.XXXX/TAP.XXXX.XXXXXXX
[2]-[5] and/or performing optimizations at several frequencies [6], [7] in the range of interest.

Regarding the analysis of reflectarrays, there are many different approaches, although the most popular, given the quasi-periodicity nature of this antenna, is the one based on a full-wave analysis assuming local periodicity [1], [8][10]. This kind of approach uses the Floquet theorem and it embeds the reflectarray unit cell in an infinite array comprised of the same unit cell, and it is illuminated by an oblique incident plane wave, taking approximately into account mutual coupling and substrate losses [8]-[10]. Although it is common in the literature to implement such an analysis with the Method of Moments, as in [8]-[13], the use of other full-wave analysis techniques such as Finite Differences in Time Domain (FDTD) [14], [15] or Finite Element Method (FEM) [16], [17] is also possible. Full wave analysis of the whole antenna can be done with different methods (e.g., FEM [18], MoM [19] or others [20]), but due to the large amounts of memory and computing time involved, they may not be of practical use for antenna design or optimization.

Reflectarray full-wave analysis based on local periodicity presents a good trade-off between accuracy and computing time, and has been widely used to analyze and design antennas with very tight requirements for space applications [1], [6], [7]. Very recently, reflectarray optimization using a fullwave analysis based on local periodicity has demonstrated a reasonable computational performance [21]. Nonetheless, other optimization approaches, relying on the use of Artificial Neural Networks (ANNs) [22], [23] or databases [11], are faster, although at the expense of losing accuracy in the unit cell model. One of the reasons for the use of full-wave analysis techniques based on local periodicity is the accurate prediction of the crosspolar pattern. Since this component of the far field is several orders of magnitude lower than the copolar pattern, high accuracy in the computation of the reflection coefficient matrix, which fully characterizes the behavior of the unit cell [1], is needed.

In order to accelerate the reflectarray analysis and optimization, a unit cell model based on ANNs [22]-[30] or databases [11], [31]-[34] may be used. The main disadvantages of databases are the high number of samples required and the use of interpolation. The latter produces a loss of accuracy, specially in the cross-coefficients of the reflection coefficient matrix, whose magnitude is very low compared to 
the direct coefficients [1]. The ANN obtains a model for the electromagnetic behavior of the cell and it is free from the accuracy loss associated to the interpolation. Nevertheless, this approach may suffer from overfitting [35]. In addition, most works related to ANNs have only produced models dealing with phases of the direct coefficients [22]-[27], sometimes also including the magnitude of a direct coefficient [28], [30], and usually only taking into account one polarization [23]-[26], [28], [30], which limits the applications of the designed reflectarrays with that technique. In [22], [27] dualpolarized reflectarrays are considered, but only the phase of the reflection coefficients are modeled. Finally, limited results have been obtained in the cross-coefficients [29], [30] and crosspolar far field when ANNs are used for the prediction of the full reflection coefficient matrix. Up to the knowledge of the authors, [29] is the only work dealing with dual-polarized reflectarrays and the full matrix of reflection coefficients using ANNs.

In this work, we propose the use of Support Vector Machines (SVMs) [36] as an alternative to the use of databases or ANNs in order to obtain an accurate model of the full reflection coefficient matrix to efficiently analyze linear, dualpolarized reflectarray antennas. SVMs improve ANNs since they overcome the ANNs limitation of overfitting and require less training patterns to obtain accurate results, thus taking less time in obtaining a more accurate model. In order to accelerate the SVM training and improve its accuracy, strategies to speed up computations and reduce the number of input variables to the SVM are detailed. The SVM model is tested against a set of different test patterns, produced by the MoM-LP, obtaining accurate results for all coefficients of the reflection coefficient matrix. Finally, the SVM model is used in the analysis of reflectarray antennas, comparing the obtained radiation patterns with those of a MoM-LP simulation, obtaining a high degree of agreement for both copolar and crosspolar patterns in both linear polarizations, while greatly speeding up the analysis computing time.

This paper is organized as follows. Section II provides a brief overview of the SVMs theory and reflectarray analysis. Section III details the strategies followed for the SVM training in order to increase accuracy and speed up computations. Section IV shows the comparison between the SVM model and MoM-LP simulation for different reflection coefficients and the analysis of the whole antenna comparing the radiation patterns. Finally, Section V contains the conclusions.

\section{SVM THEORY AND REFLECTARRAY ANALYSIS}

\section{A. SVM Theoretical background}

SVMs are automatic learning structures that are able to deal with both classification and regression problems [37]. In this work, the regression problem is adapted to find a model that best fits the behavior of a reflectarray unit cell for a given set of inputs. SVMs belong to a class of supervised learning in which a training set, that is a set of inputs $\left(\vec{x}_{i}\right)$ and outputs $\left(y_{i}\right)$, is used to estimate a function $f$ that relates inputs and outputs. Then, the estimated function $f$ is employed to associate each new input to its corresponding output. Formally speaking, given a training set $T=\left\{\left(\vec{x}_{i}, y_{i}\right)\right\}_{i=1,2, \ldots, N_{r}}, \vec{x}_{i} \in \chi \subseteq$ $\mathbb{R}^{L}, y_{i} \in \mathbb{R}$, it is possible to find a mathematical function $f: \chi \rightarrow \mathbb{R}$, such that the output for a new element $\vec{x}$ that belongs to the input space $(\chi)$ is estimated as follows:

$$
\widetilde{y}=f(\vec{x}),
$$

where $\widetilde{y}$ is the SVM estimation of the actual output $y$ corresponding to $\vec{x}$.

The regression function $f$ may be nonlinear. In such cases, the SVM maps the input space into a high-dimensional space, called feature space $(\mathcal{H})$, where the regression may be accurately performed using a linear function. Feature space and input space are the same when $f$ is linear. Assuming that the training data (mapped, if necessary, to $\mathcal{H}$ ) is properly fitted to a linear function, the statistical learning theory states that the optimal regression function is maximally flat [37]. In this case, optimality means that $f$ has good generalization properties, i.e., that it will properly fit data of the same nature but not initially included in the training set. To obtain a maximally flat function that properly fits the training data, it is suggested to minimize the regularized risk functional, $R_{\text {reg }}[f]$, which considers the empirical errors and the flatness of $f$ :

$$
R_{\mathrm{reg}}[f] \propto \frac{\|\vec{w}\|^{2}}{2}+C \sum_{i=1}^{N_{r}} c\left(\vec{x}_{i}, y_{i}, f\left(\vec{x}_{i}\right)\right),
$$

where $\vec{w}$ is a weight vector in $\mathcal{H},\|\cdot\|$ stands for the Euclidean norm, and $\|\vec{w}\|^{2} / 2$ acts as a regularization term whose minimization assures to provide flatness of $f$ in $\mathcal{H}$; $c(\vec{x}, y, f(\vec{x}))$ is the cost (or loss) function associated to the regression error and $C$ is a positive constant that determines a trade-off between minimizing the empirical risk (regression error) and providing good generalization properties [38]. The introduction of slack variables to the regression problem leads to a constrained minimization problem whose solution is a function $f$ of the form:

$$
f(\vec{x})=\langle\vec{w}, \vec{\phi}(\vec{x})\rangle+b=\sum_{i=1}^{N_{s}}\left[\left(\alpha_{i}^{-}-\alpha_{i}^{+}\right) K\left(\vec{x}_{i}, \vec{x}\right)\right]+b,
$$

where $\vec{x}_{i}$ are the support vectors; $N_{s}$ is the number of support vectors; $\alpha_{i}^{-}$and $\alpha_{i}^{+}$are the optimal Lagrange multipliers; $b$ is the offset, and $K\left(\vec{x}_{i}, \vec{x}\right)$ is the kernel function that, operating with arguments in the input space $\left(\vec{x}_{i}, \vec{x}\right)$, produces the dot product of their images in the feature space, i.e.: $K\left(\vec{x}_{i}, \vec{x}\right)=$ $\left\langle\vec{\phi}\left(\vec{x}_{i}\right), \vec{\phi}(\vec{x})\right\rangle$.

In this work, we use the $\epsilon$-insensitive loss function since it provides a sparse representation of function $f$ in terms of the support vectors, i.e. it requires a smaller value of $N_{s}$ than other loss functions [37]. The $\epsilon$-insensitive loss function is defined as follows:

$$
c(\vec{x}, y, f(\vec{x}))=\max \{0,|y-f(\vec{x})|-\epsilon\} .
$$

Note that the cost function defined in (4) does not penalize regression errors below a given $\epsilon \geq 0$. Therefore, the parameter $\epsilon$, that must be chosen beforehand, is related to the maximum accuracy that the SVM model may achieve. In addition, we 
use a Gaussian Kernel (also known as Radial Basis Function, $\mathrm{RBF}$ ) which follows the equation

$$
K\left(\vec{x}, \vec{x}^{\prime}\right)=\exp \left(-\gamma\left\|\vec{x}-\vec{x}^{\prime}\right\|^{2}\right),
$$

where $\gamma$ is a tunable parameter that may be interpreted as the inverse of double the variance of the Gaussian function. Equation (3) expresses function $f$ as a linear combination of versions of the kernel function plus an offset. A low value of $\gamma$ yields a mixture of wide Gaussian functions and, very likely, a soft function $f$. On the other hand, a high value of $\gamma$ produces a mixture of narrow Gaussian functions and, very likely, a very rich function $f$. As a consequence, the optimal value of $\gamma$ depends on the shape of the function to be estimated.

From the above description it is clear that the SVM model is defined by the parameters $C, \gamma$ and $\epsilon$. Furthermore, the proper selection of these parameters is of fundamental importance since, for a given problem, they are related to the model accuracy.

\section{B. Reflectarray analysis}

Assuming a single-offset reflectarray configuration [1] (see Fig. 1), the primary feed imposes an incident electric field on the reflectarray surface. The reflected electric field can be computed at each reflectarray element $(m, n)$ as follows:

$$
\vec{E}_{\mathrm{ref}}^{X / Y}\left(x_{m}, y_{n}\right)=\mathbf{R}^{m n} \cdot \vec{E}_{\mathrm{inc}}^{X / Y}\left(x_{m}, y_{n}\right),
$$

where the superscript indicates the antenna polarization, $\left(x_{m}, y_{n}\right)$ are the coordinates of the $(m, n)$ th element and

$$
\mathbf{R}^{m n}=\left(\begin{array}{cc}
\rho_{x x}^{m n} & \rho_{x y}^{m n} \\
\rho_{y x}^{m n} & \rho_{y y}^{m n}
\end{array}\right),
$$

is the reflection coefficient matrix which relates both fields at each element. The components of matrix $\mathbf{R}^{m n}$ are complex numbers and fully characterize the reflectarray unit cell. $\rho_{x x}^{m n}$ and $\rho_{y y}^{m n}$ are known as the direct coefficients while $\rho_{x y}^{m n}$ and $\rho_{y x}^{m n}$ are the cross-coefficients. An accurate characterization of the cross-coefficients, as well as the direct coefficients, is necessary to achieve a correct prediction of the crosspolar far field [21]. To that end, matrix $\mathbf{R}^{m n}$ is computed with a full-wave analysis tool assuming local periodicity. This task is the most time consuming operation when analyzing a reflectarray. Hence, in order to substantially speed up the reflectarray analysis, a fast computation of $\mathbf{R}^{m n}$ is required. This acceleration may be achieved using SVMs to obtain a surrogate model of $\mathbf{R}^{m n}$. To the best of the authors' knowledge, most noted efforts to speed up the computation of $\mathbf{R}^{m n}$ have been focused on the fast characterization of the phases of the direct coefficients [22]-[25] though not on the complete reflection coefficient matrix.

Finally, once the tangential reflected field has been obtained with (6), the radiation patterns can be efficiently computed using the Fast Fourier Transform algorithm, and the copolar and crosspolar far field components obtained using Ludwig's third definition of crosspolarization [21] for linear polarization.

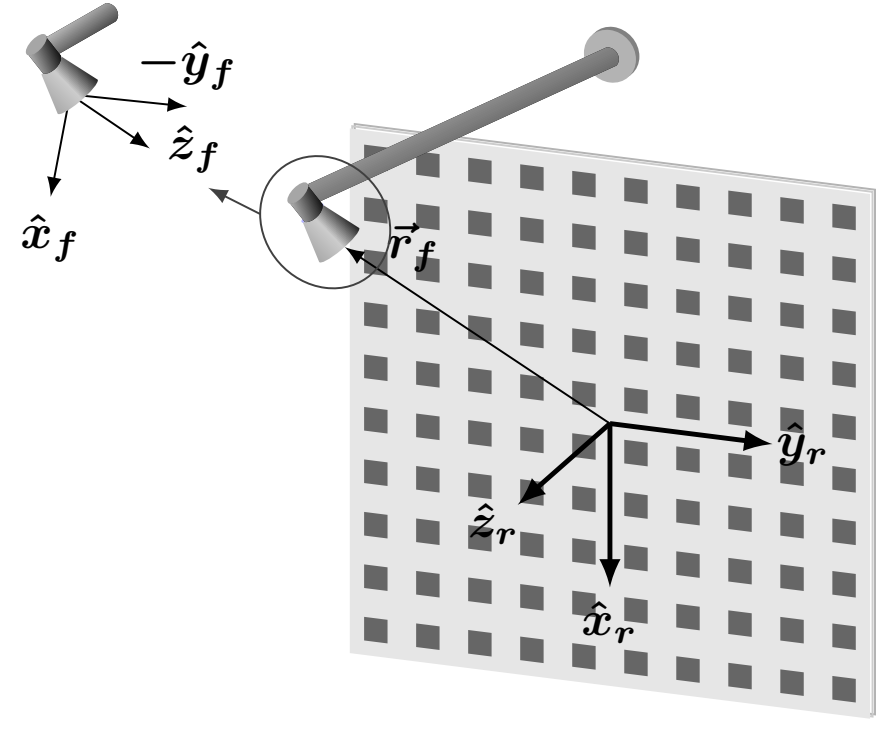

Fig. 1. Sketch of the reflectarray single-offset geometry.

\section{Characterization of Unit Cells Using SVM}

\section{A. Input space and input parameters}

The considered reflectarray unit cell is shown in Fig. 2. It is comprised of eight parallel and coplanar dipoles in two layers of metallization. Each polarization is controlled by a set of four dipoles and both sets are shifted half a unit cell's period from each other. The goal of the SVM training is to obtain a surrogate model of the reflection coefficient matrix in (7). To obtain the training samples for the SVM, $\mathbf{R}^{m n}$ is computed using the spectral domain MoM-LP presented in [9], which has been validated with measurements of prototypes [39]-[41], waveguide measurements to simulate periodic conditions [42], full-wave simulations of the whole antenna [43] and full-wave simulations assuming local periodicity [42], [44].

This matrix depends on several variables, such as: frequency, angle of the impinging plane wave (incident angle), geometry specifications (periodicity, length and width of the dipoles, and separation between them, see Fig. 2), and substrate properties. Therefore, the unit cell offers many degrees of freedom, with multiple resonances where the function exhibits a highly non-linear behavior, and the number of those resonances grows with the number of input variables. Since the SVM model should accurately predict $\mathbf{R}^{m n}$ for any value of those input variables, in this paper we propose to reduce the number of variables to generate the surrogate model.

We initially fix the substrate and the periodicity. The substrate is usually set to the values of some commercially available substrate. For the present case, the CuClad 233 substrate is used for both layers, with a thickness of $0.787 \mathrm{~mm}$ and a complex relative permittivity $\varepsilon_{r}=2.33-j 3.029 \cdot 10^{-3}$. To maintain fixed the physical dimensions of the array, we employ a unit cell size of $5.84 \mathrm{~mm} \times 5.84 \mathrm{~mm}$, which is approximately half a wavelength at the frequency of analysis, which is set to $25.5 \mathrm{GHz}$. In addition, we also consider a reduction on the geometry specifications of the unit cell. For the element employed in this work, the phase shift for the 


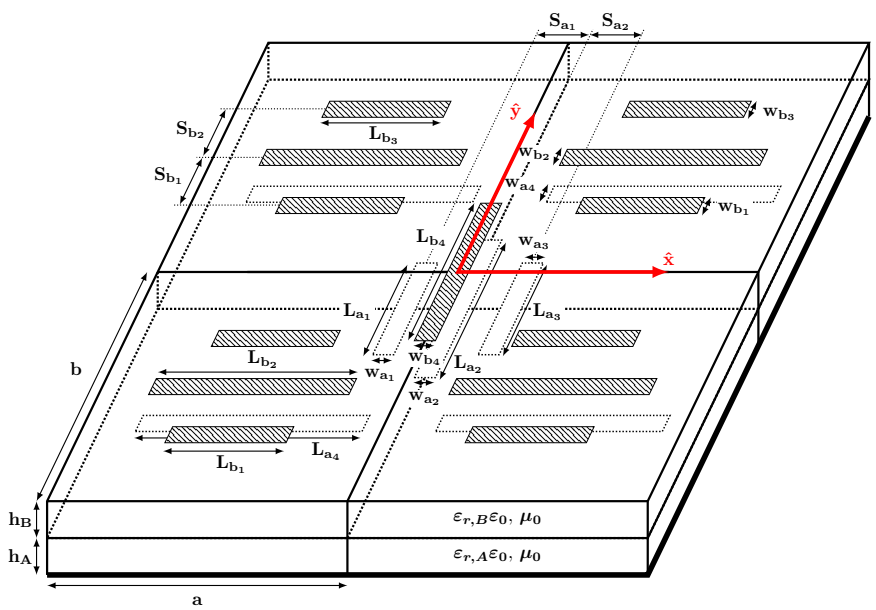

Fig. 2. Employed reflectarray unit cell based on parallel and coplanar dipoles in two layers of metallization.

reflectarray design is obtained by varying the dipole lengths (see [4], [39]), thus the dipole widths and the separation between dipoles may be fixed without a noticeable loss in performance. In this work, we set the dipole width to $0.5 \mathrm{~mm}$ and the separation between dipoles to $4 \mathrm{~mm}$.

The incident angle $(\theta, \varphi)$ of the impinging plane wave on each reflectarray element depends on the position of the element. An accurate analysis of the reflectarray requires to take this fact into account. In this work, a different surrogate model is obtained for each incident angle, and thus the incident angle is treated as an input parameter and not as an input variable to produce the SVM model.

Even though the input variables have been reduced to eight dipole lengths, a further complexity reduction would facilitate the SVM training process. In this regard, two geometrical variables are defined, $T_{x}$ and $T_{y}$, and each one controls the dipole lengths for each polarization. The variables $T_{x}$ and $T_{y}$ are considered to be defined over the interval $[0.1,5] \mathrm{mm}$; thus, the input space is $\chi=[0.1,5] \mathrm{mm} \times[0.1,5] \mathrm{mm}$. The relation between the dipole lengths and these new variables are as follows (see Fig. 2):

$$
\begin{aligned}
& L_{a_{4}}=T_{x} ; L_{b_{1}}=L_{b_{3}}=0.63 T_{x} ; L_{b_{2}}=0.93 T_{x}, \\
& L_{b_{4}}=0.95 T_{y} ; L_{a_{1}}=L_{a_{3}}=0.58 T_{y} ; L_{a_{2}}=T_{y} .
\end{aligned}
$$

In summary, $T_{x}$ and $T_{y}$ are the input variables of the SVM, and an SVM model is obtained for each value of the input parameters. Finally, despite the proposed reduction in the number of degrees of freedom of the unit cell shown in Fig. 2, it is worth mentioning that shaped beams with high performances may be obtained in practice by optimizing just one or two reflectarray geometrical parameters [11].

\section{B. Output variables}

The reflection coefficients of (7) are complex numbers and SVMs are conceived to estimate real valued functions. Thus, at least eight models must be trained for each incident angle. Initially, it is best to train the real and imaginary parts instead of the magnitude and phase since it is not possible to provide an accurate unwrapped version of the phase over a randomly distributed set of points in $\chi$. The obtention of the magnitude and the phase of the reflection coefficients is straightforward from their real and imaginary parts.

However, the shape of the direct coefficients magnitude is very soft and close to unity, while the shape of their real and imaginary parts fluctuate in a more striking way. As a result, the estimation of $\left|\rho_{x x}\right|$ and of $\left|\rho_{y y}\right|$ is much more accurate if these variables are trained directly than if they are computed from the estimation of the real and imaginary parts. This behavior is not extensive to the cross-coefficients. Therefore, we consider ten output variables for each incident angle: $\left|\rho_{x x}\right|,\left|\rho_{y y}\right|, \operatorname{Re}\left\{\rho_{x x}\right\}, \operatorname{Im}\left\{\rho_{x x}\right\}, \operatorname{Re}\left\{\rho_{x y}\right\}, \operatorname{Im}\left\{\rho_{x y}\right\}$, $\operatorname{Re}\left\{\rho_{y x}\right\}, \operatorname{Im}\left\{\rho_{y x}\right\}, \operatorname{Re}\left\{\rho_{y y}\right\}$, and $\operatorname{Im}\left\{\rho_{y y}\right\}$.

\section{Model selection and training}

The LIBSVM library [38] with the Gaussian kernel of (5) is used to get the SVM model. The main goal of the SVM model selection is to find the optimal parameters $(C, \gamma)$, noted as $\left(C^{*}, \gamma^{*}\right)$, that are supposed to minimize the relative error of the estimation over a set of data that were not initially included in the training set. The error function is defined as follows:

$$
e(\vec{x}, y, f(\vec{x}))=y-f(\vec{x}),
$$

where $f(\vec{x})$ is the output of the SVM model and $y$ is the actual output of the MoM-LP.

Lets assume that the regression, for a given SVM model, is computed over a set of $M$ input samples $\vec{x}_{i}, i=1,2, \ldots, M$. Consequently, a set of output samples $\left(y_{i}\right)$, a set of samples of function $f\left(f\left(\vec{x}_{i}\right)\right)$, and the corresponding set of samples of error function $\left(e_{i}=e\left(\vec{x}_{i}, y_{i}, f\left(\vec{x}_{i}\right)\right)\right)$ are produced. In this manner, we may define the vectors of samples: $\vec{y}=$ $\left(y_{1}, y_{2}, \ldots, y_{M}\right)$ and $\vec{e}=\left(e_{1}, e_{2}, \ldots, e_{M}\right)$. To obtain a measurement of the accuracy of the function estimation over the set of $M$ samples, we use the relative error that is defined by

$$
R E=\frac{\|\vec{e}\|}{\|\vec{y}\|}=\frac{\sqrt{\sum_{i=1}^{M}\left|e_{i}\right|^{2}}}{\sqrt{\sum_{i=1}^{M}\left|y_{i}\right|^{2}}},
$$

or equivalently, its logarithmic value in $\mathrm{dB}$ as follows

$$
10 \log \left(R E^{2}\right)=10 \log \left(\frac{\|\vec{e}\|^{2}}{\|\vec{y}\|^{2}}\right),
$$

that expresses the quotient between the energy of the error vector $\vec{e}$ and the energy of the output vector $\vec{y}$. This way, a small error produces an approximation function $f$ that is close to the reference function $y$ without regard to the function being close to 0 (cross-coefficients) or close to 1 (direct coefficients), since the error is referenced to the corresponding output samples. The selected optimal parameters $\left(C^{*}, \gamma^{*}\right)$ should minimize (10) and (11).

In addition to the selection of the parameters $\left(C^{*}, \gamma^{*}\right)$, as indicated in a previous section, it is necessary to choose the parameter $\epsilon$. The size of the insensitive zone $(\epsilon)$ is related to the maximum achievable precision on the function estimation 
by the SVM model. In fact, all the samples of the function that fall inside the $\epsilon$-tube (or the insensitive zone) produce no contribution to the loss function of (4), though they actually contribute to the regression error of (10). Assuming that, asymptotically, all the training samples, except the support vectors, fall within the $\epsilon$-tube and that the samples of the error function are uniformly distributed in the interval $[-\epsilon,+\epsilon]$, it is possible to estimate the variance of the error function samples by $\sigma_{e}^{2}=E\left\{|e|^{2}\right\}=\epsilon^{2} / 3$. Then, using (10), the variance of the $R E$ over a set of $N_{r}$ training samples is calculated as

$$
\sigma_{R E}^{2}=E\left\{R E^{2}\right\}=\frac{N_{r} \sigma_{e}^{2}}{\|\vec{y}\|^{2}} .
$$

Therefore, given a set of $N_{r}$ samples and a desired accuracy, expressed in terms of $\sigma_{R E}^{2}$, the value of $\epsilon$ turns out to be

$$
\epsilon \approx \sqrt{\frac{3}{N_{r}}} \sigma_{R E}\|\vec{y}\|
$$

In practice, eq. (12) represents a lower threshold for the quadratic value of the relative error that is only achieved for a sufficiently large $N_{r}$ (in principle, $N_{r} \rightarrow \infty$ ). As a consequence, the approximation of $\epsilon$ given by (13) is more accurate the larger $N_{r}$ is. Thus, a proper value of $N_{r}$ is also necessary to achieve a desired accuracy. The selection of $N_{r}$ for the problem at hand is discussed in a following section.

The selection of the optimal parameters $\left(C^{*}, \gamma^{*}\right)$ is not a trivial question and it is very dependent on the problem under study. It is actually not possible to make an adequate choice beforehand. Hence, it is necessary to use a trial and error procedure. In this work, we use the cross-validation procedure to select $\left(C^{*}, \gamma^{*}\right)$. In this procedure, the whole data set, composed by $N$ samples, is divided into three non-overlapping subsets: training $\left(\leq 70 \%\right.$, i.e. $\left.N_{r} \leq 0.7 N\right)$, validation $(15 \%$, i.e. $\left.N_{v}=0.15 N\right)$ and test $\left(15 \%\right.$, i.e. $\left.N_{t}=0.15 N\right)$. For a given value of $(C, \gamma)$, the training set is used to train the SVM, i.e. to obtain the SVM model. The produced surrogate model is evaluated over the validation set and the $R E$ over this set, associated to $(C, \gamma)$, is computed. This procedure is performed over a limited size grid on the $(C, \gamma)$ plane, usually about a few tenths (in logarithm to base 2) above and below 0 for both $C$ and $\gamma$. The optimum parameters $\left(C^{*}, \gamma^{*}\right)$ are selected as those which minimize the $R E$ over the validation set. Finally, the error of the selected optimum model is evaluated with the test set to estimate the error produced on new samples. This process ensures that the sets employed to compute the error during the grid search and to test the final model remain hidden during the training process and are not actual training samples, so the generalization properties of the model are better accounted for.

In the following, $C_{2}=\log _{2} C$ and $\gamma_{2}=\log _{2} \gamma$ to alleviate notation. Fig. 3 shows the relative error (in $\mathrm{dB}$ ) of the SVM models generated with different values of $\left(C_{2}, \gamma_{2}\right)$ for the estimation of $\operatorname{Re}\left\{\rho_{y x}\right\}$ and incidence $\left(\theta=20^{\circ}, \varphi=20^{\circ}\right)$. In this case, the number of samples is $N_{r}=1750, N_{v}=$ $N_{t}=375$ and the step considered for both $C_{2}$ and $\gamma_{2}$ is $\Delta C_{2}=\Delta \gamma_{2}=0.2$. The grid search yields $C_{2}^{*}=10.4$, $\gamma_{2}^{*}=5.4$ and $10 \log \left(R E^{2}\right)=-36.05 \mathrm{~dB}$, over the validation set. This search, or model selection, took $50006 \mathrm{~s}$ (about $13 \mathrm{~h}$

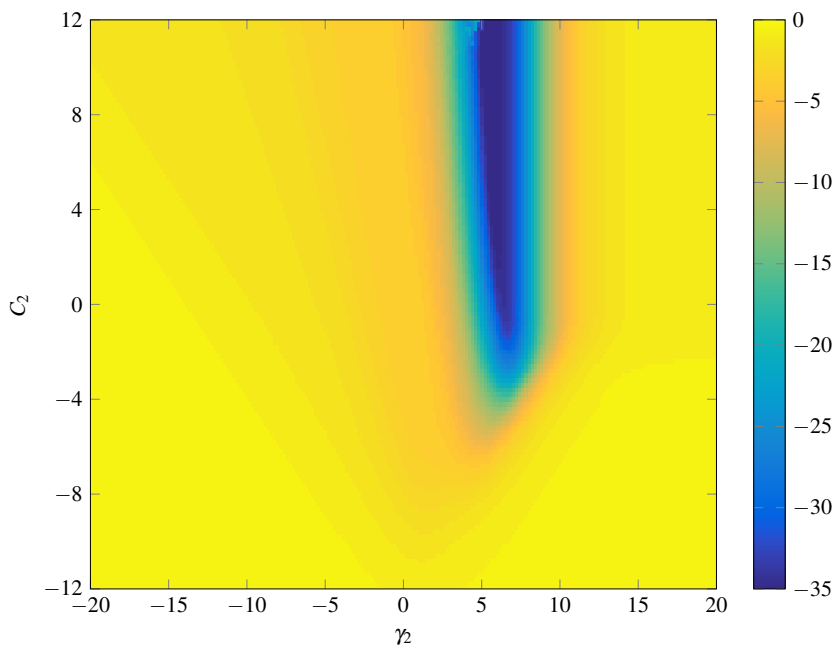

Fig. 3. Relative error (in $\mathrm{dB}$ ) over the validation set for an exhaustive grid search in the $\left(C_{2}, \gamma_{2}\right)$ plane with $\Delta C_{2}=\Delta \gamma_{2}=0.2$ and estimating $\operatorname{Re}\left\{\rho_{y x}\right\}$ for incident angle $\left(\theta=20^{\circ}, \varphi=20^{\circ}\right)$.

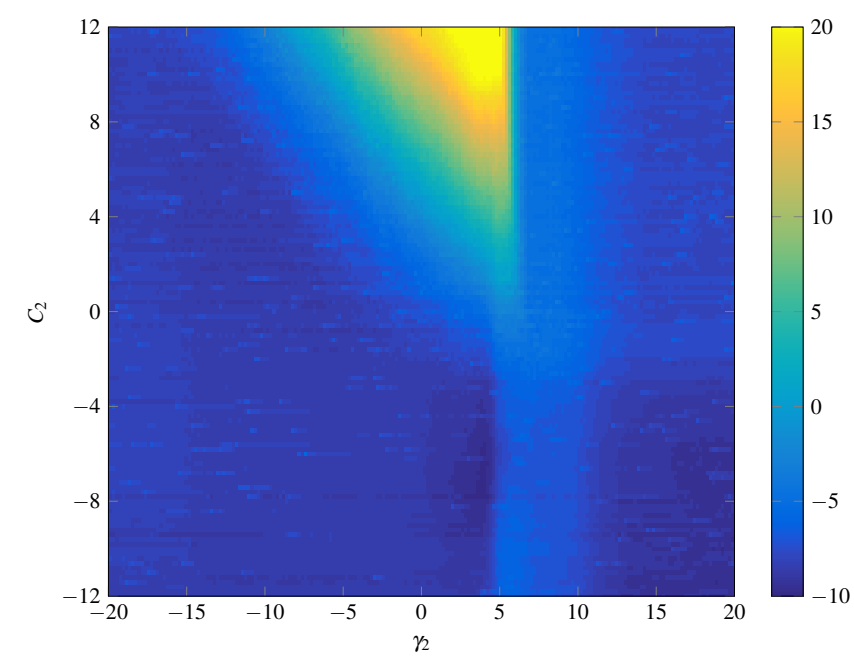

Fig. 4. Training time in logarithmic units $\left(10 \log \left(t_{\text {train }}[\mathrm{s}]\right)\right)$ for each point of the grid search of Fig. 3 .

and $54 \mathrm{~min}$ ). Considering that we estimate 10 output variables per incident angle and that the number of incident angles may be substantial, this brings to light the necessity of using an efficient grid search.

\section{Efficient grid search}

In this section, we detail a strategy to speed up the search of the optimal model $\left(C_{2}^{*}, \gamma_{2}^{*}\right)$. The first approach to reduce the model selection time is based on the observation of the shape of the $R E$ in the $\left(C_{2}, \gamma_{2}\right)$ plane. The shape of the $R E$ is very similar for different output parameters and incident angles and it is characterized by a narrow valley along $C_{2}$, as shown in Fig. 3. In addition, Fig. 4 plots the time cost of the training associated to each point on the $\left(C_{2}, \gamma_{2}\right)$ grid of Fig. 3. This plot shows that the training time cost for large values of $C_{2}$ in the area of interest may be three orders of magnitude higher than for smaller values of $C_{2}$. Taking all this 


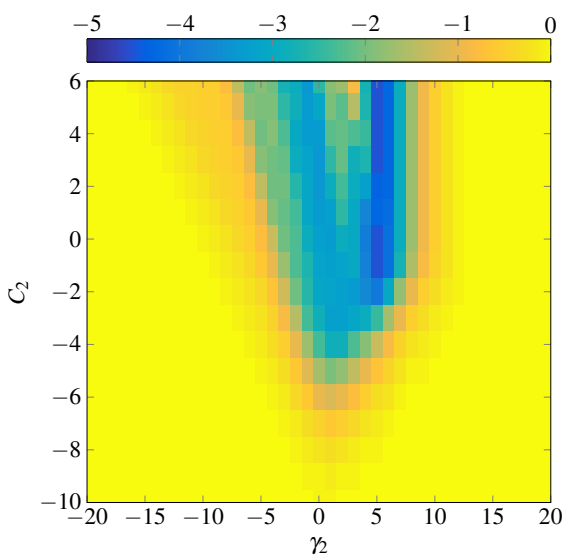

(a)

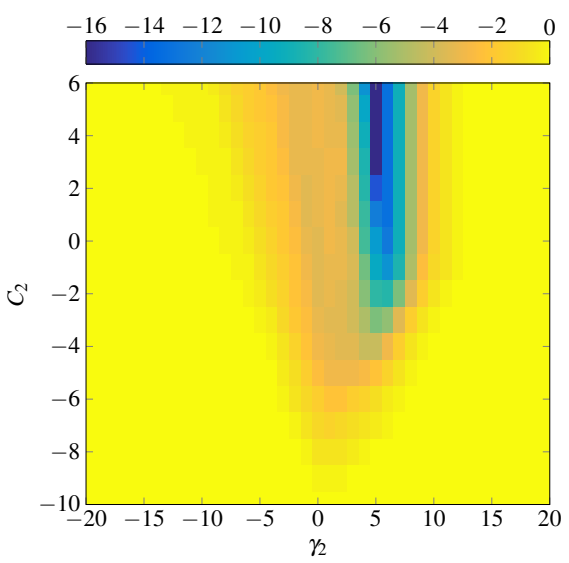

(b)

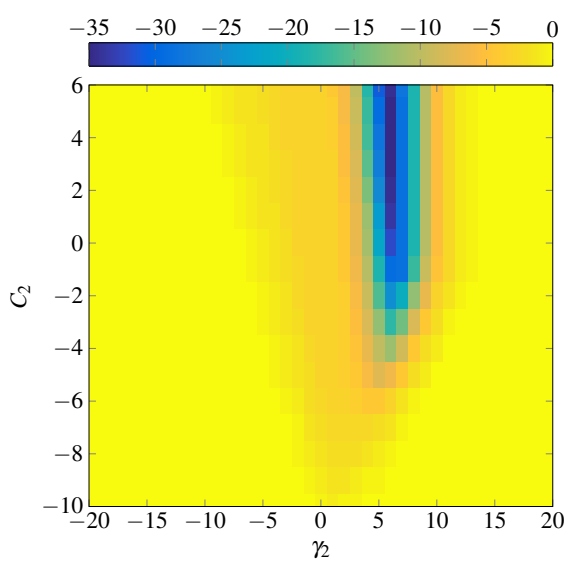

(c)

Fig. 5. Relative error (in dB) over the validation set $\left(N_{v}=375\right)$ for an exhaustive grid search in the $\left(C_{2}, \gamma_{2}\right)$ plane with $\Delta C_{2}=\Delta \gamma_{2}=1$, estimating $\operatorname{Im}\left\{\rho_{x y}\right\}$ for oblique incidence $\left(\theta=10^{\circ}, \varphi=20^{\circ}\right)$ and using (a) $5 \%$, (b) $20 \%$ and (c) $100 \%$ samples of the training pattern set $\left(N_{r}=1750\right)$.

into consideration, we have decided to limit the grid search to the domain $\gamma_{2} \in[-20,+20]$ and $C_{2} \in[-10,+6]$.

Another way of reducing the grid search time cost is reducing the number of training samples. Fig. 5 shows the relative error on the $\left(C_{2}, \gamma_{2}\right)$ grid for the estimation of $\operatorname{Im}\left\{\rho_{x y}\right\}$ with incidence $\left(\theta=10^{\circ}, \varphi=20^{\circ}\right)$ and using three different training set sizes $\left(N_{r}=87,350,1750\right)$. In this case, the considered step for both $C_{2}$ and $\gamma_{2}$ is $\Delta C_{2}=\Delta \gamma_{2}=1$ and the search took $1.2 \mathrm{~s}$ for $N_{r}=87,10.0 \mathrm{~s}$ for $N_{r}=350$, and $196.4 \mathrm{~s}$ for $N_{r}=1750$. As it can be observed, the valley where the optimum is located shifts its position and becomes deeper, though the region where it appears is approximately the same. Note that the scale is intentionally different for each case to clearly place the valley. Therefore, a grid search with a reduced number of samples (note the great resemblance between Figs. 5(b) and 5(c)) may yield a good starting point to get $\left(C_{2}^{*}, \gamma_{2}^{*}\right)$ at an affordable time cost.

In order to obtain an accurate estimation of $\left(C_{2}^{*}, \gamma_{2}^{*}\right)$ it is possible to use a local optimizer starting at a point that is near to it. We use the Nelder-Mead simplex method [45] as local optimizer due to its computational performance. The NelderMead method is classified as a direct search method since it tries to minimize a scalar-valued non-linear function using only function values, without derivative information. At each iteration, it keeps a simplex of $L+1$ vertices, where $L$ is the dimensionality of the problem. In the present case, $L=2$ and the simplex is a triangle. At each iteration, the number of function evaluations can be 1,2 or $L+2$, depending on the steps taken by the algorithm [46]. In practice, the most common steps only perform 1 or 2 evaluations of the function, with the steps performing $L+2$ evaluations being very rare [46]. Given the relative error as a function of $\left(C_{2}, \gamma_{2}\right)$ and a suitable starting point, this optimization algorithm would take few function evaluations to reach the minimum.

Sometimes, the Nelder-Mead method may stagnate on a local minimum whose associated $R E$ is higher than expected. In those cases, it is necessary to re-initiate the algorithm on another, better starting point. Moreover, the grid search performed with a reduced number of samples (see Fig. 5), may give a starting point to the Nelder-Mead method that drives it to one of those undesired local minima. This drawback may be overcome by performing a grid search in the vicinity of the minimum which was obtained by the search with the reduced number of samples, but this time considering all the training samples before the Nelder-Mead proceeds. In addition, it is also of interest that the Nelder-Mead method avoids searching on large values of $C_{2}$ since those values considerably slow down the error function evaluation. Since the Nelder-Mead method does not allow to perform a bounded search, we have used a variant of this method ${ }^{1}$ that inserts a wrapper function around the relative error function which permits to accomplish a bounded search. In this particular case, we have imposed that $C_{2} \leq 6$.

The suggested grid search is as follows:

1) Perform a wide grid search, using $20 \%$ of the training samples, over $\gamma_{2} \in[-20,+20]$ and $C_{2} \in[-10,+6]$ with $\Delta C_{2}=\Delta \gamma_{2}=1$. The value of $\left(C_{2}, \gamma_{2}\right)$ where the minimum $R E$ on the validation set is achieved at this stage is noted as $\left(C_{2}^{1)}, \gamma_{2}^{1)}\right)$.

2) Perform a localized grid search, using all the training samples and the same resolution than in 1), over the proximity of $\left(C_{2}^{1)}, \gamma_{2}^{1)}\right)$. The value of $\left(C_{2}, \gamma_{2}\right)$ where the minimum $R E$ on the validation set is achieved at this stage is noted as $\left(C_{2}^{2)}, \gamma_{2}^{2)}\right)$.

3) If $\left(C_{2}^{2)}, \gamma_{2}^{2)}\right)$ provides the desired accuracy on the validation data, then approximate the optimal parameters as $\left(C_{2}^{*}=C_{2}^{2)}, \gamma_{2}^{*}=\gamma_{2}^{2)}\right)$.

Otherwise, use the training data to run the Nelder-Mead algorithm with starting point at $\left(C_{2}^{2)}, \gamma_{2}^{2)}\right)$ and impose $C_{2} \leq 6$ to find $\left(C_{2}^{*}, \gamma_{2}^{*}\right)$ that minimizes the validation error.

Finally, the model error is obtained with the test set at $\left(C_{2}^{*}, \gamma_{2}^{*}\right)$. Using this strategy the model selection time for the first example (shown in Figs. 3 and 4 ) has been reduced

\footnotetext{
${ }^{1}$ Matlab function fminsearchbnd.m by John D’Errico
} 


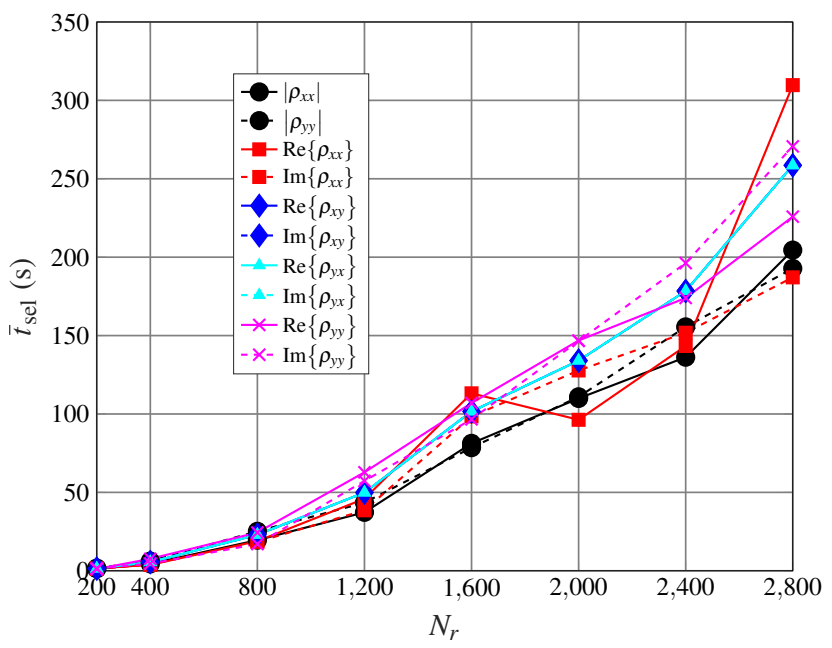

Fig. 6. Evolution of the average model selection time with the number of training patterns.

from $50006 \mathrm{~s}$ to $65 \mathrm{~s}$. The efficient grid search technique yields $C_{2}^{*}=3.8096$ and $\gamma_{2}^{*}=5.7188$ and $10 \log \left(R E^{2}\right)=$ $-35.32 \mathrm{~dB}$, over the validation set. Thus, this technique reduces the time cost of the exhaustive grid search about three orders of magnitude while it provides a slight increase of the relative error (less than one $\mathrm{dB}$ in the present example).

\section{E. Discussion on the number of training patterns, time cost and achieved error}

The grid search strategy detailed in the previous section to obtain $\left(C^{*}, \gamma^{*}\right)$ yields values of $C$ and $\gamma$ close to the optimal ones with a limited time cost. Nonetheless, it is interesting to study the influence of the number of training patterns on the achieved accuracy and error.

On the one hand, the model selection time cost $\left(t_{\text {sel }}\right)$ increases with the number of training patterns. Fig. 6 shows the average time cost $\left(\bar{t}_{\mathrm{sel}}\right)$ of the proposed grid search strategy for every estimated output variable of the SVM model. This average has been performed over all the considered incident angles. On the other hand, the relative error decreases with the number of training patterns. Fig. 7 plots the average value, over all the considered incident angles, of the relative error $(\overline{R E})$ in $\mathrm{dB}$ over the test set for every estimated output variable. It shows that the error rapidly decreases with the number of training patterns until approximately $1600-2000$ patterns, where the slope of the relative error starts to approach to zero. As a consequence, from Figs. 6 and 7 it seems that a good trade-off between training time and relative error may be achieved for $N_{r} \in[1600,2000]$. In this work, we have decided to use $N_{r}=1750$. Nonetheless, it must be taken into consideration that this number depends on the problem to solve. It is also possible to observe that the achieved relative error for the magnitude of both direct coefficients is almost the same (asymptotically tending to $-80 \mathrm{~dB}$ ) and that the relative error for the real and imaginary parts of all the reflection coefficients is also very similar (asymptotically tending to $-40 \mathrm{~dB}$ ). Note that $\sigma_{R E}^{2}$ was set to $-40 \mathrm{~dB}$ for the real and imaginary parts and to $-80 \mathrm{~dB}$ for the magnitude of direct
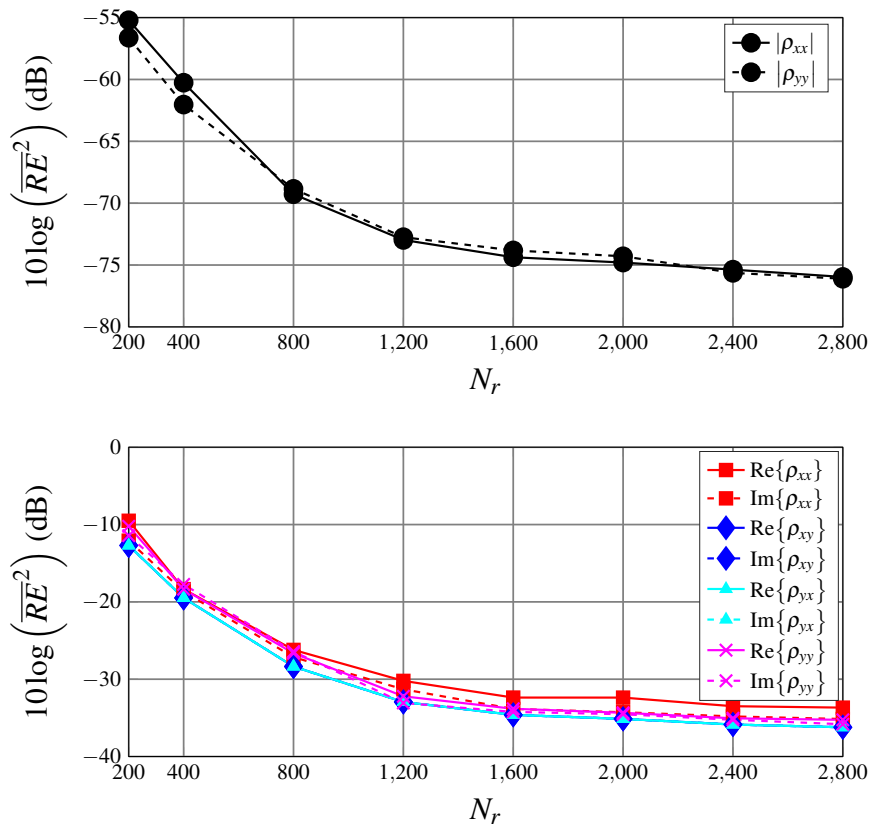

Fig. 7. Variation of the average relative error over the test set with the number of training patterns.

coefficients, and that using (13) the value of $\epsilon$ is adaptively computed as a function of the Euclidean norm of the output training data vector $(\|\vec{y}\|)$, for a given value of $N_{r}$.

Table I summarizes the results for both model selection time cost and relative error over the test set, for all the estimated output variables when using $N_{r}=1750, N_{v}=375$, and $N_{t}=375$. All the results given in this section have been obtained using, in sequential mode, a workstation with 2 Intel Xeon E5-2650v3 CPU at $2.3 \mathrm{GHz}$ and $256 \mathrm{~GB}$ of RAM. It is worth mentioning that the visible reduction of the time cost registered on the table values compared to the plot in Fig. 6 is because the simulations of the table strictly follow the grid search strategy given in Section III.D, while the simulations related to the plots do not avoid the Nelder-Mead method even when the SVM model with parameters $\left(C_{2}^{2)}, \gamma_{2}^{2)}\right)$ yields the desired accuracy.

\section{Antenna Analysis Results}

\section{A. Antenna specifications}

A single-offset reflectarray configuration is considered (see Fig. 1). The antenna is rectangular and is comprised of 900 elements in a regular grid of $30 \times 30$ elements. As primary feed, a horn modeled as a $\cos ^{q} \theta$ function is employed [47], with an illumination taper of $-19.5 \mathrm{~dB}$ at the reflectarray edges. The feed phase center is placed at $(-94,0,214) \mathrm{mm}$ in the reflectarray coordinate system [1] and points to the reflectarray center. The considered unit cell is the one described in Section III and shown in Fig. 2. The working frequency is $25.5 \mathrm{GHz}$ and the periodicity is $5.84 \mathrm{~mm} \times 5.84 \mathrm{~mm}$, which is approximately half a wavelength. In addition, the incident angles are discretized in $10^{\circ}$ steps on $\theta$ and $\varphi$, as shown in Fig. 8, in order to reduce the number of SVM models. A total of $57(\theta, \varphi)$ incident angles are considered, although only 31 
Table I

AVERAGE RELATIVE ERROR OVER THE TEST SET AND AVERAGE MODEL SELECTION TIME OF THE BEST SVM MODEL FOR EACH OUTPUT VARIABLE.

\begin{tabular}{ccccccccccc}
\hline Variable & $\left|\rho_{x x}\right|$ & $\left|\rho_{y y}\right|$ & $\operatorname{Re}\left\{\rho_{x x}\right\}$ & $\operatorname{Im}\left\{\rho_{x x}\right\}$ & $\operatorname{Re}\left\{\rho_{x y}\right\}$ & $\operatorname{Im}\left\{\rho_{x y}\right\}$ & $\operatorname{Re}\left\{\rho_{y x}\right\}$ & $\operatorname{Im}\left\{\rho_{y x}\right\}$ & $\operatorname{Re}\left\{\rho_{y y}\right\}$ & $\operatorname{Im}\left\{\rho_{y y}\right\}$ \\
\hline $10 \log \left(\overline{R E}^{2}\right)(\mathrm{dB})$ & -74.4 & -74.4 & -32.9 & -33.7 & -35.4 & -33.4 & -33.7 & -35.2 & -34.4 & -34.8 \\
$\bar{t}_{\text {sel }}(\mathrm{s})$ & 72.4 & 80.2 & 48.0 & 55.6 & 71.8 & 71.8 & 71.8 & 71.8 & 79.6 & 75.0 \\
\hline
\end{tabular}

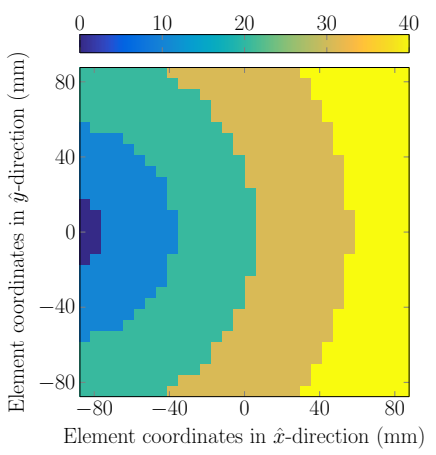

(a)

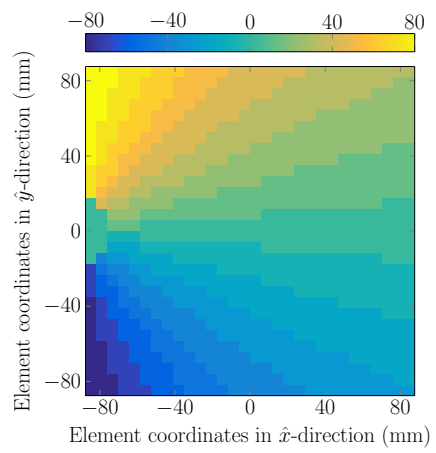

(b)
Fig. 8. Discretization of the incident angles in (a) $\theta$ and (b) $\varphi$ in $10^{\circ}$ steps.

of them are used to train the SVM, and the rest of SVMs are constructed using symmetries. Since 10 SVM models per angle are considered (magnitude of the direct coefficients, real and imaginary parts of the four reflection coefficients), a total of 310 SVM models will be trained for this reflectarray. In addition, the training of each reflection coefficient for each incident angle is independent from the rest, so it can be easily parallelized, further saving computing time.

\section{B. Reflection coefficients}

Once all the SVM models have been obtained, and in order to assess the accuracy of the models, simulations of the reflection coefficients with the SVM model and MoMLP will be compared. Fig. 9 shows the results in phase for the two reflection coefficients with the largest test error: $\rho_{x x}$ for $\left(\theta=40^{\circ}, \varphi=0^{\circ}\right)$ and $\rho_{x y}$ for $\left(\theta=30^{\circ}, \varphi=60^{\circ}\right)$. As it can be seen, the difference between the two curves, provided by MoM-LP and SVM, is very small. The highest discrepancies occur for the cross-coefficient $\rho_{x y}$ where there are phase jumps. Nevertheless, the abrupt phase changes are followed by the SVM model and the mean absolute error is $1.17^{\circ}$ for the phase of $\rho_{x y}$ and $0.39^{\circ}$ for $\rho_{x x}$. On the other hand, Fig. 10 shows the magnitude for the same cut and the same coefficients. As it can be seen, even though the cross-coefficient presents a highly non-linear behavior, the SVM model matches with very high accuracy the MoM-LP simulation, including the amplitude resonances.

Furthermore, the SVM model was compared to MoM-LP simulations in the whole $\left(T_{x}, T_{y}\right)$ grid, and they show a high degree of agreement, similar to the results shown in Figs. 9 and 10, for all coefficients. From the two coefficients shown in Figs. 9 and 10, $\rho_{x y}$ for $\left(\theta=30^{\circ}, \varphi=60^{\circ}\right)$ presents the highest test error, both in real and imaginary parts, and they

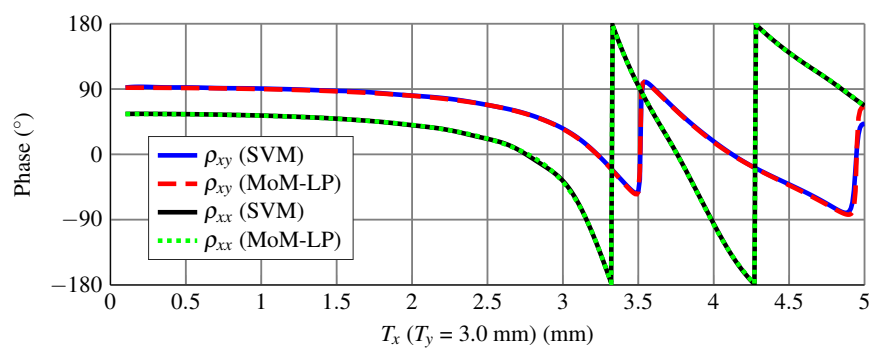

(a)

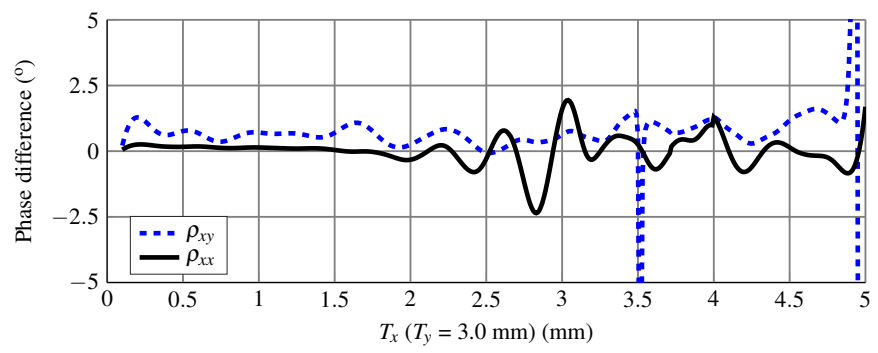

(b)

Fig. 9. Comparison of $\rho_{x x}\left(\theta=40^{\circ}, \varphi=0^{\circ}\right)$ and $\rho_{x y}\left(\theta=30^{\circ}, \varphi=60^{\circ}\right)$ between SVM and MoM-LP as a function of $T_{x}$ for the cut $T_{y}=3 \mathrm{~mm}$ in (a) phase and (b) phase difference in degrees between SVM and MoM-LP. These two coefficients present the highest test error for a direct and crosscoefficient.

are shown in Fig. 11 in the $\left(T_{x}, T_{y}\right)$ plane with a step of $0.05 \mathrm{~mm}$ in both dimensions. The coefficient was simulated using MoM-LP, and the SVM needs to fit those functions. On the other hand, Fig. 12 shows the phase of $\rho_{x y}$, both for SVM and MoM-LP simulations. For the MoM-LP case, the phase was calculated using the data from Fig. 11. As it can be seen, the SVM predicts the shape of the phase distribution with a high degree of accuracy, in particular all the $180^{\circ}$ jumps, which correspond with minima in the amplitude of the crosscoefficients (as observed in the amplitude and phase of $\rho_{x y}$ in Figs. 9 and 10), and can be used in reflectarray design to lower the crosspolar pattern [48], [49].

\section{Radiation patterns}

The final goal of using the SVM to model the reflectarray unit cell is to efficiently analyze the antenna to accurately predict the radiation patterns. Three test cases are provided here for the same antenna optics: a pencil beam pointing at $\left(\theta=5.4^{\circ}, \varphi=0^{\circ}\right)$, an isoflux pattern for global Earth coverage [50] and a shaped pattern for Local Multipoint Distribution Service central station with a squared-cosecant pattern in elevation and sectored-beam pattern in azimuth [51]. For all cases, three analyses were performed, one with the 


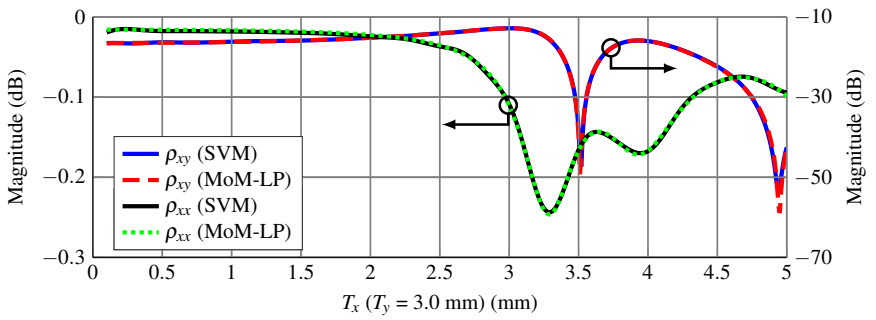

(a)

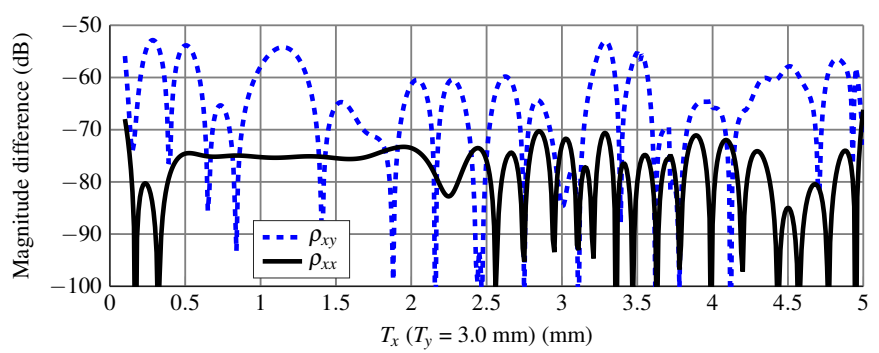

(b)

Fig. 10. Comparison of $\rho_{x x}\left(\theta=40^{\circ}, \varphi=0^{\circ}\right)$ and $\rho_{x y}\left(\theta=30^{\circ}, \varphi=60^{\circ}\right)$ between SVM and MoM-LP as a function of $T_{x}$ for the cut $T_{y}=3 \mathrm{~mm}$ in (a) magnitude and (b) magnitude difference in dB between SVM and MoMLP. These two coefficients present the highest test error for a direct and crosscoefficient.

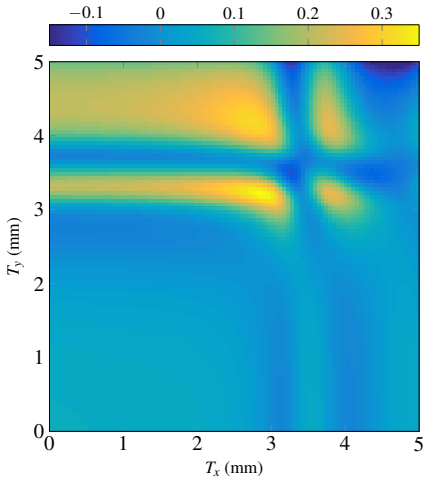

(a)

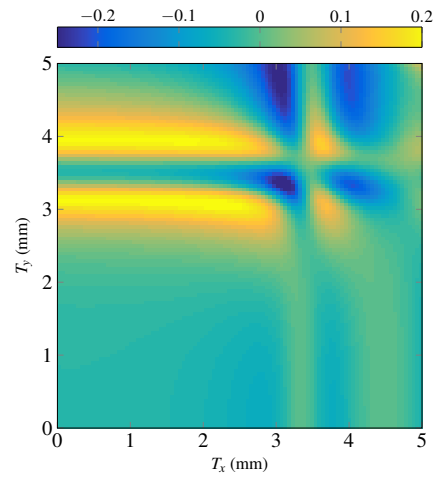

(b)
Fig. 11. (a) Real and (b) imaginary parts of $\rho_{x y}$ for $\left(\theta=30^{\circ}, \varphi=60^{\circ}\right)$ simulated with MoM-LP. This coefficient presents the highest test error both in real and imaginary parts after the SVM model is obtained.

SVM and two with MoM-LP considering the actual angles of incidence from the feed at each reflectarray element, and another with the same discretization of the angles of incidence shown in Fig. 8, which is the same used by the SVM.

Fig. 13 shows the comparison between MoM-LP and SVM in predicting the pencil beam radiation pattern for $\mathrm{Y}$ polarization. As it can be seen, the copolar pattern is accurately predicted by the SVM, including the secondary lobes and all the nulls. For the crosspolar pattern, the agreement is also very good in the regions where the crosspolar presents the maximum value. However, there are some discrepancies for lower values of the crosspolar pattern when comparing the SVM simulation with MoM-LP considering the real angles of incidence. Nevertheless, these differences are due to the discretization employed in the SVM (shown in Fig. 8), and they appear at pattern levels $60 \mathrm{~dB}$ below the maximum value.

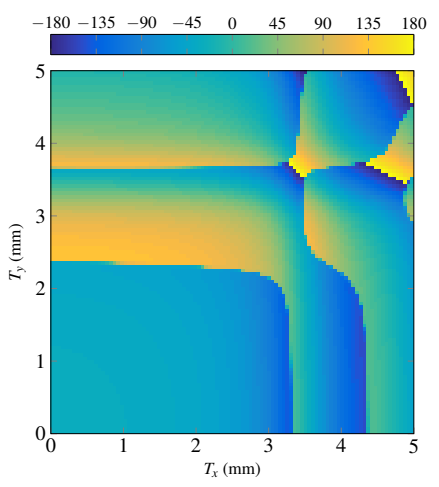

(a)

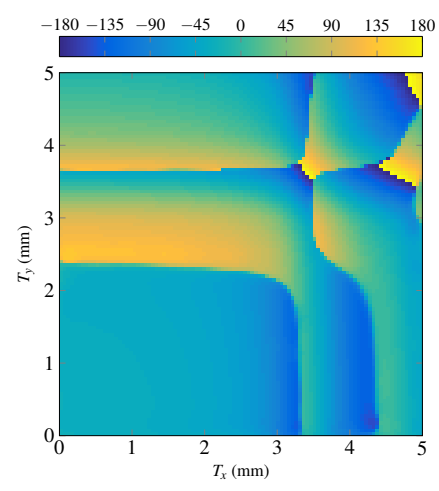

(b)
Fig. 12. Comparison of the phase of $\rho_{x y}$ for $\left(\theta=30^{\circ}, \varphi=60^{\circ}\right)$ obtained with (a) MoM-LP and (b) SVM in the whole $\left(T_{x}, T_{y}\right)$ plane. This coefficient presents the highest test error both in real and imaginary parts.

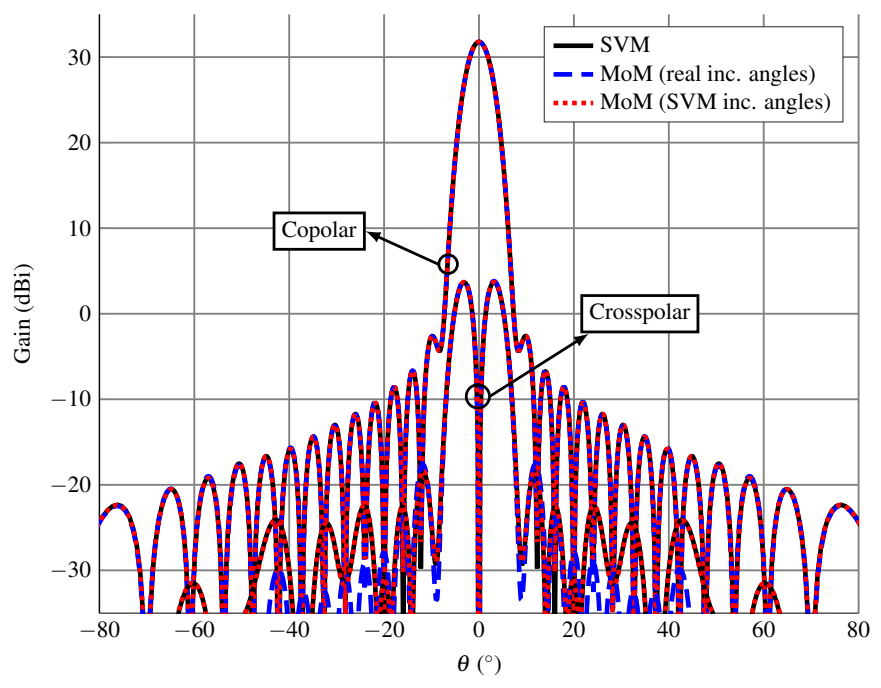

Fig. 13. Copolar and crosspolar main cut $\left(\varphi=90^{\circ}\right)$ for a pencil beam pattern comparing MoM-LP and SVM simulations for Y polarization.

When the same discretization is used by SVM and MoM-LP, the crosspolar pattern is accurately predicted.

Figs. 14 and 15 show the main cuts for the copolar and crosspolar components for the isoflux and LMDS patterns, respectively, for $\mathrm{Y}$ polarization. Again, both copolar and crosspolar patterns are accurately predicted, as well as all the nulls and side lobes when the same discretization of the incidence angles is used. In all cases, the prediction of the copolar pattern is more robust against this discretization, which mainly affects the crosspolar pattern for regions outside its maximum value. If higher accuracy is desired for the crosspolar pattern in those regions, a finer discretization than the one shown in Fig. 8 should be employed.

All the results shown were produced for $\mathrm{Y}$ polarization, but similar results and conclusions were obtained for $\mathrm{X}$ polarization. Overall, the agreement between the MoM-LP simulation and the SVM model is very high for both copolar and crosspolar patterns, as demonstrated by the results of the three radiation patterns for both linear polarizations. In addition, all three patterns were compared in the whole visible 


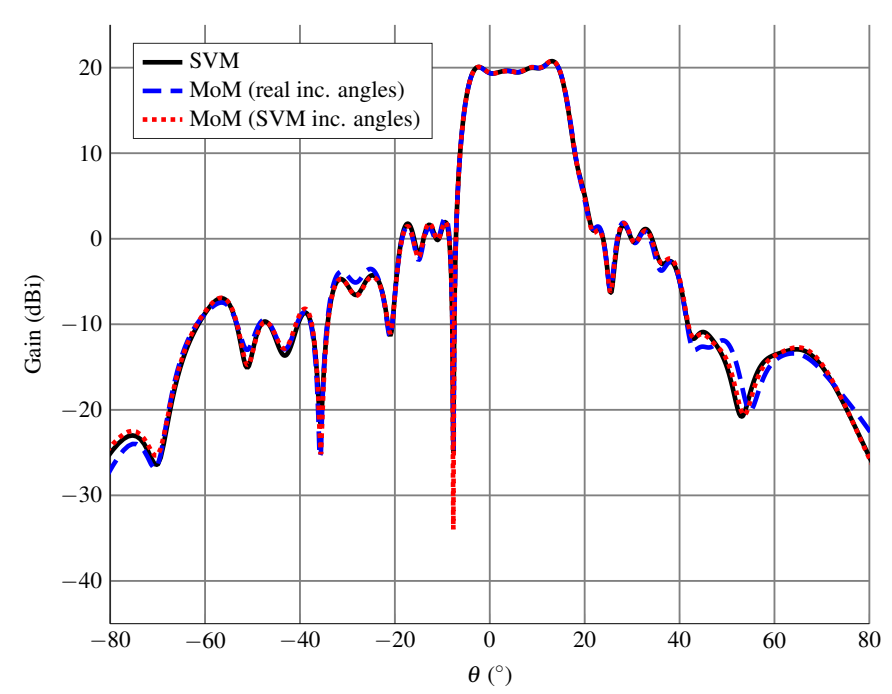

(a)

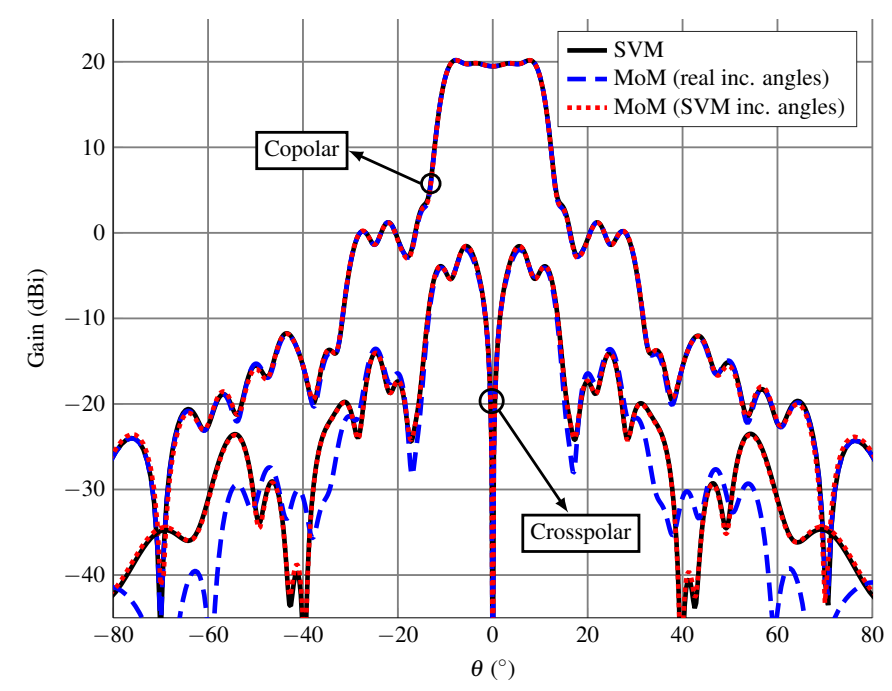

(b)

Fig. 14. Main cuts of the copolar and crosspolar patterns (Y polarization) for an isoflux pattern for global Earth coverage comparing simulations of the MoM-LP and SVM. (a) $\varphi=0^{\circ}$. (b) $\varphi=90^{\circ}$.

region, computing the relative error between the SVM and MoM-LP simulations according to:

$$
R E=\frac{\left\|E_{\mathrm{MoM}}-E_{\mathrm{SVM}}\right\|}{\left\|E_{\mathrm{MoM}}\right\|}
$$

where $\|\cdot\|$ denotes the Euclidean norm and $E$ either the copolar or the crosspolar pattern. The results are shown in Table II, where the error (14) is shown in percentage, the analysis was performed with the same incident angles for MoM-LP and SVM, and the radiation patterns were computed with a resolution of $512 \times 512$ points, but only those belonging to the visible region were used in computing the error. As it can be seen, the errors are very low due to the high accuracy obtained in the SVM models.

Finally, the reflectarray analysis is accelerated by a factor larger than three orders of magnitude, specifically, a factor larger than 2000 was obtained using the SVM in comparison with MoM-LP. Tests were carried out in an Intel Core i3-

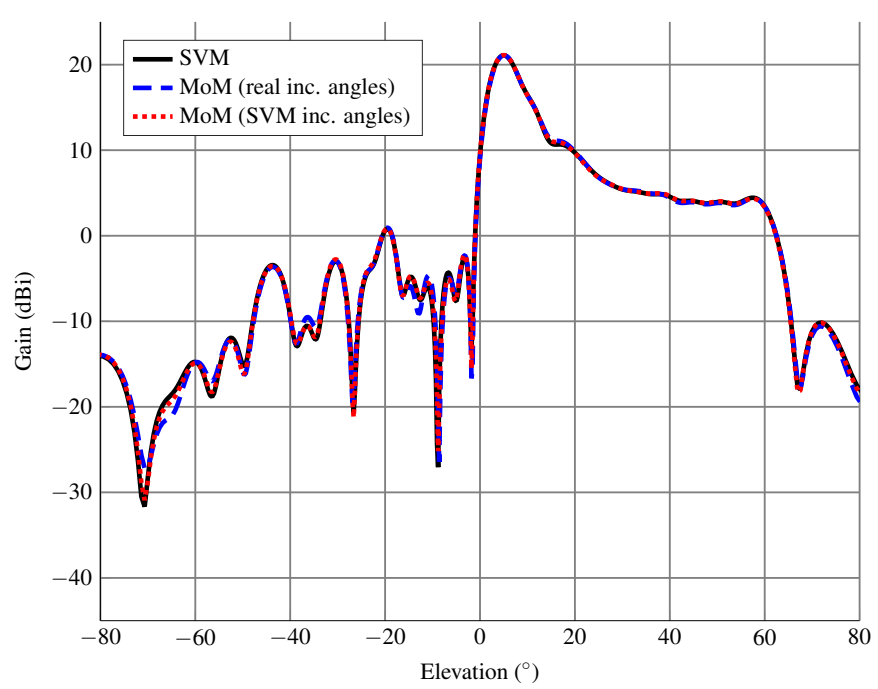

(a)

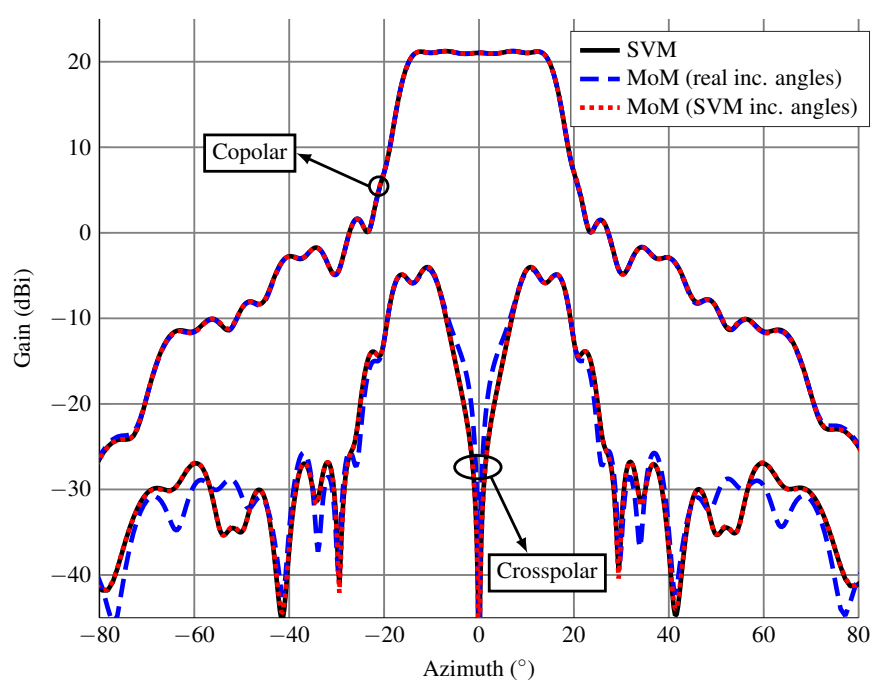

(b)

Fig. 15. Main cuts of the copolar and crosspolar patterns ( $Y$ polarization) for LMDS application in (a) elevation (azimuth at $0^{\circ}$ ) and (b) azimuth (elevation at $5.4^{\circ}$ ), comparing simulations of the MoM-LP and SVM.

2100 with $4 \mathrm{CPU}$ (two physical and two virtual) at $3.1 \mathrm{GHz}$, where the 900-element reflectarray took a mean time of $84.5 \mathrm{~s}$ to be analyzed with MoM-LP, while it took only $0.031 \mathrm{~s}$ with the SVM, when analyzed in single-threaded mode (gain factor of 2725). When the analysis was parallelized using OpenMP [52], those times were $22.3 \mathrm{~s}$ for MoM-LP and $0.0085 \mathrm{~s}$ for the SVM (gain factor of 2623). As comparison, some ANN implementations have been reported to be between 200 [27] and 700 [22] times faster than MoM-LP, although only computing the phases of the direct coefficients, which saves some computational burden with regard to the computation of the full matrix. Regarding memory usage, the SVM requires more than MoM-LP since many SVM models need to be loaded, one per output variable and angle of incidence. In the example considered, while the MoM-LP tool used around $600 \mathrm{~KB}$ of memory, the SVM used roughly $27 \mathrm{MB}$. In addition, if more SVM models were employed, the memory requirements would increase. Nevertheless, the memory requirement for the SVM 
Table II

RELATIVE ERROR BETWEEN THE RADIATION PATTERNS PREDICTED BY MOM-LP AND THE SVM FOR BOTH POLARIZATIONS.

\begin{tabular}{ccccc}
\hline & \multicolumn{2}{c}{ Pol. X } & \multicolumn{2}{c}{ Pol. Y } \\
\cline { 2 - 5 } & CP & XP & CP & XP \\
\hline Pencil & $0.20 \%$ & $0.61 \%$ & $0.36 \%$ & $0.75 \%$ \\
LMDS & $0.51 \%$ & $1.07 \%$ & $0.57 \%$ & $1.18 \%$ \\
Isoflux & $0.43 \%$ & $1.32 \%$ & $0.56 \%$ & $0.36 \%$ \\
\hline
\end{tabular}

is negligible if compared with other data structures used in array optimization [21], [53], and nowadays it is not an issue even in personal computers.

Once the surrogate model is obtained, it can be employed in design and optimization processes instead of the MoMLP routine, saving considerably more time than the required to obtain the model. Furthermore, if high accuracy is needed for the crosspolar optimization of reflectarray antennas, the SVM may be trained with a finer discretization of the incident angles. Moreover, the SVM model can be used in the first stages of the optimization, in order to obtain a suitable starting point for a more accurate optimization using MoM-LP with more degrees of freedom, as in [21], [54]. This way, the optimization process is sped up thanks to the use of SVMs, while still obtaining accurate results since a further refinement is achieved employing MoM-LP in the latest steps. In addition, the availability of an accurate and computationally fast model of the full reflection coefficient matrix by means of SVMs allows the use of global search algorithms for reflectarray crosspolar optimization within reasonable computing time, which is not currently possible using MoM-LP since the computations are very slow for such a task.

\section{CONCLUSIONS}

This paper has addressed the characterization of the full matrix of reflection coefficients by means of Support Vector Machines (SVMs) for a fast and accurate analysis of dualpolarized reflectarray antennas as an alternative to the use of Artificial Neural Networks (ANNs) and databases to overcome some of their issues. The SVM is used to obtain surrogate models of the amplitudes of the direct coefficients as well as the real and imaginary parts of the four reflection coefficients. A detailed discussion on the selection of optimal SVM parameters is provided. In addition, since the SVM training process is slow, a strategy to speed it up has been presented, which relies on a combination of the reduction of the initial number of training patterns, a localized grid search and the use of a grid-search local optimizer. It allows to improve the average training time by a factor larger than three orders of magnitude with regard to a naive exhaustive grid search. This strategy was employed to obtain the SVM models for a reflectarray unit cell comprised of two sets of four parallel dipoles in two layers of metallization. Each set controls one linear polarization, so the SVM models can be employed for the analysis of dual-polarized shaped-beam reflectarrays. The results for amplitude and phase of the reflection coefficients show excellent agreement between the Method of Moments based on Local Periodicity (MoM-LP) and SVM simulations, even for the cross-coefficients, despite their highly non-linear behavior, which is difficult to model. Finally, three different radiation patterns were considered to assess the accuracy of the new technique, a pencil beam pattern for point-to-point communications, an isoflux pattern for global Earth coverage and a shaped-beam pattern for Local Multipoint Distribution Service application. The radiation patterns were computed for both linear polarizations and the copolar and crosspolar components were compared. For the three patterns, both far field components show a high degree of agreement between the SVM and MoM-LP simulations. Moreover, the analysis is sped up by a factor larger than three orders of magnitude using the SVM instead of MoM-LP.

\section{ACKNOWLEDGMENT}

The authors would like to thank R. F. Díaz, Ph.D.; Prof. R. R. Boix, and Prof. J. A. Encinar for providing the MoMLP software to obtain training samples and carry out the simulations.

\section{REFERENCES}

[1] J. Huang and J. A. Encinar, Reflectarray Antennas. Hoboken, NJ, USA: John Wiley \& Sons, 2008.

[2] J. A. Encinar and J. A. Zornoza, "Broadband design of three-layer printed reflectarrays," IEEE Trans. Antennas Propag., vol. 51, no. 7, pp. 1662-1664, Jul. 2003.

[3] J. H. Yoon, Y. J. Yoon, W. sang Lee, and J. ho So, "Broadband microstrip reflectarray with five parallel dipole elements," IEEE Antennas Wireless Propag. Lett., vol. 14, pp. 1109-1112, 2015.

[4] R. Florencio, J. Encinar, R. R. Boix, and G. Perez-Palomino, "Dualpolarisation reflectarray made of cells with two orthogonal sets of parallel dipoles for bandwidth and cross-polarisation improvement," IET Microw. Antennas Propag., vol. 8, no. 15, pp. 1389-1397, Aug. 2014.

[5] L. Moustafa, R. Gillard, F. Peris, R. Loison, H. Legay, and E. Girard, "The phoenix cell: A new reflectarray cell with large bandwidth and rebirth capabilities," IEEE Antennas Wireless Propag. Lett., vol. 10, pp. 71-74, 2011.

[6] J. A. Encinar, L. S. Datashvili, J. A. Zornoza, M. Arrebola, M. SierraCastaner, J. L. Besada-Sanmartin, H. Baier, and H. Legay, "Dualpolarization dual-coverage reflectarray for space applications," IEEE Trans. Antennas Propag., vol. 54, no. 10, pp. 2827-2837, Oct. 2006.

[7] J. A. Encinar, M. Arrebola, L. F. de la Fuente, and G. Toso, "A transmitreceive reflectarray antenna for direct broadcast satellite applications," IEEE Trans. Antennas Propag., vol. 59, no. 9, pp. 3255-3264, Sep. 2011.

[8] C. Wan and J. A. Encinar, "Efficient computation of generalized scattering matrix for analyzing multilayered periodic structures," IEEE Trans. Antennas Propag., vol. 43, no. 11, pp. 1233-1242, Nov. 1995.

[9] R. Florencio, R. R. Boix, and J. A. Encinar, "Enhanced MoM analysis of the scattering by periodic strip gratings in multilayered substrates," IEEE Trans. Antennas Propag., vol. 61, no. 10, pp. 5088-5099, Oct. 2013.

[10] D. M. Pozar and T. A. Metzler, "Analysis of a reflectarray antenna using microstrip patches of variable size," Electron. Lett., vol. 29, no. 8, pp. 657-658, Apr. 1993.

[11] M. Zhou, S. B. Sørensen, O. S. Kim, E. Jørgensen, P. Meincke, and O. Breinbjerg, "Direct optimization of printed reflectarrays for contoured beam satellite antenna applications," IEEE Trans. Antennas Propag., vol. 61, no. 4, pp. 1995-2004, Apr. 2013.

[12] D. Gonzalez-Ovejero, F. Mesa, and C. Craeye, "Accelerated macro basis functions analysis of finite printed antenna arrays through 2D and 3D multipole expansions," IEEE Trans. Antennas Propag., vol. 61, no. 2, pp. 707-717, Feb. 2013.

[13] O. M. Bucci, A. Capozzoli, G. D’Elia, and S. Musto, "A new approach to the power pattern synthesis of reflectarrays," in Proc. URSI International Symposium on Electromagnetic Theory (EMTS'04), Pisa, Italy, May 2327, 2004, pp. 1053-1055. 
[14] R. T. Lee and G. S. Smith, "An alternative approach for implementing periodic boundary conditions in the FDTD method using multiple unit cells," IEEE Trans. Antennas Propag., vol. 54, no. 2, pp. 698-705, Feb. 2006.

[15] E. Girard, R. Moulinet, R. Gillard, and H. Legay, “An FDTD optimization of a circularly polarized reflectarray unit cell," in IEEE Antennas and Propagation Society International Symposium, vol. 3, San Antonio, Texas, USA, Jun. 16-21, 2002, pp. 136-139.

[16] R. Chiniard, A. Barka, and O. Pascal, "Hybrid FEM/Floquet modes/PO technique for multi-incidence RCS prediction of array antennas," IEEE Trans. Antennas Propag., vol. 56, no. 6, pp. 1679-1686, Jun. 2008.

[17] V. de la Rubia, J. Zapata, and M. A. González, "Finite element analysis of periodic structures without constrained meshes," IEEE Trans. Antennas Propag., vol. 56, no. 9, pp. 3020-3028, Sep. 2008.

[18] M. Rütschlin, T. Wittig, and Z. Iluz, "Phased antenna array design with CST Studio Suite," in $10^{\text {th }}$ European Conference on Antennas and Propagation (EuCAP), Davos, Switzerland, Apr. 10-15, 2016, pp. 1-5.

[19] I. González, A. Tayebi, J. Gomez, C. Delgado, and F. Cátedra, "Fast analysis of a dual-band reflectarray using two different numerical approaches based on the moment method," IEEE Trans. Antennas Propag., vol. 61, no. 4, pp. 2333-2336, Apr. 2013.

[20] P. De Vita, A. Freni, F. Vipiana, P. Pirinoli, and G. Vecchi, "Fast analysis of large finite arrays with a combined multiresolution-SM/AIM approach," IEEE Trans. Antennas Propag., vol. 54, no. 12, pp. 38273832, Dec. 2006.

[21] D. R. Prado, M. Arrebola, M. R. Pino, R. Florencio, R. R. Boix, J. A. Encinar, and F. Las-Heras, "Efficient crosspolar optimization of shaped-beam dual-polarized reflectarrays using full-wave analysis for the antenna element characterization," IEEE Trans. Antennas Propag., vol. 65, no. 2, pp. 623-635, Feb. 2017.

[22] P. Robustillo, J. Zapata, J. A. Encinar, and J. Rubio, "ANN characterization of multi-layer reflectarray elements for contoured-beam space antennas in the Ku-band," IEEE Trans. Antennas Propag., vol. 60, no. 7, pp. 3205-3214, Jul. 2012.

[23] D. Caputo, A. Pirisi, M. Mussetta, A. Freni, P. Pirinoli, and R. E. Zich, "Neural network characterization of microstrip patches for reflectarray optimization," in $3^{\text {rd }}$ European Conference on Antennas and Propagation (EuCAP), Berlin, Germany, Mar. 23-27, 2009, pp. 2520-2522.

[24] S. Nesil, F. Güneş, and U. Özkaya, "Phase characterization of a reflectarray unit cell with minkowski shape radiating element using multilayer perceptron neural network," in $7^{\text {th }}$ International Conference on Electrical and Electronics Engineering (ELECO), vol. 2, Bursa, Turkey, Dec. 1-4, 2011, pp. 219-222.

[25] H. M. Linh, M. Mussetta, P. Pirinoli, and R. E. Zich, "Modeling of reflectarray elements by means of metaPSO-based artificial neural network," in $7^{\text {th }}$ European Conference on Antennas and Propagation (EuCAP), Gothenburg, Sweden, Apr. 8-12, 2013, pp. 3450-3451.

[26] F. Güneş, S. Nesil, and S. Demirel, "Design and analysis of Minkowski reflectarray antenna using 3-D CST Microwave Studio-based neural network model with particle swarm optimization," Int. J. RF Microw. Comput. Eng., vol. 23, no. 2, pp. 272-284, Mar. 2013.

[27] P. Robustillo, J. Zapata, J. A. Encinar, and M. Arrebola, "Design of a contoured-beam reflectarray for a eutelsat european coverage using a stacked-patch element characterized by an artificial neural network," IEEE Antennas Wireless Propag. Lett., vol. 11, pp. 977-980, 2012.

[28] A. Freni, M. Mussetta, and P. Pirinoli, "Neural network characterization of reflectarray antennas," Int. J. Antennas Propag., vol. 2012, pp. 1-10, May 2012.

[29] P. Robustillo, J. Zapata, J. A. Encinar, R. Florencio, R. R. Boix, and J. R. Mosig, "Accurate characterization of multi-resonant reflectarray cells by artificial neural networks," in The $8^{\text {th }}$ European Conference on Antennas and Propagation (EUCAP), The Hague, The Netherlands, Apr. 6-11, 2014, pp. 2297-2299.

[30] V. Richard, R. Loison, R. Gillard, H. Legay, and M. Romier, "Loss analysis of a reflectarray cell using ANNs with accurate magnitude prediction," in $11^{\text {th }}$ European Conference on Antennas and Propagation (EuCAP), Paris, France, Mar. 19-24, 2017, pp. 2402-2405.

[31] A. Tayebi, J. Gómez, I. González, and F. Cátedra, "Computer tool for designing reflectarray antennas," in $15^{\text {th }}$ International Symposium on Antenna Technology and Applied Electromagnetics (ANTEM), Toulouse, France, Jun. 25-28, 2012, pp. 1-4.

[32] Y. Abdallah, C. Menudier, M. Thevenot, and T. Monediere, "Synthesis of reflectarrays with mutual couplings," in $7^{\text {th }}$ European Conference on Antennas and Propagation (EuCAP), Gothenburg, Sweden, Apr. 8-12, 2013, pp. 3446-3450.

[33] A. Capozzoli, C. Curcio, A. Liseno, M. Migliorelli, and G. Toso, "Fast analysis and database generation in aperiodic reflectarrays," in $7^{\text {th }}$ Euro- pean Conference on Antennas and Propagation (EuCAP), Gothenburg, Sweden, Apr. 8-12, 2013, pp. 3913-3916.

[34] E. Ercil, L. Alatan, and O. A. Civi, "An efficient numerical solution method for reflectarrays of varying element sizes," IEEE Trans. Antennas Propag., vol. 63, no. 12, pp. 5668-5676, Dec. 2015.

[35] R. G. Ayestarán, M. F. Campillo, and F. Las-Heras, "Multiple support vector regression for antenna array characterization and synthesis," IEEE Trans. Antennas Propag., vol. 55, no. 9, pp. 2495-2501, Sep. 2007.

[36] V. Vapnik, The Nature of Statistical Learning Theory, 2nd ed. New York, NY, USA: Springer, 1999.

[37] B. Schölkopf and A. J. Smola, Learning with Kernels, 1st ed. Cambridge, Massachusetts: The MIT Press, 2001.

[38] C.-C. Chang and C.-J. Lin, "LIBSVM: A library for support vector machines," ACM Trans. Intell. Syst. Technol., vol. 2, no. 3, pp. 27:1-27:27, Apr. 2011, software available at http://www.csie.ntu.edu. tw/ cjlin/libsvm.

[39] R. Florencio, J. A. Encinar, R. R. Boix, V. Losada, and G. Toso, "Reflectarray antennas for dual polarization and broadband telecom satellite applications," IEEE Trans. Antennas Propag., vol. 63, no. 4, pp. 1234-1246, Apr. 2015.

[40] E. M. de Rioja, J. A. Encinar, M. Barba, R. Florencio, R. R. Boix, and V. Losada, "Dual polarized reflectarray transmit antenna for operation in Ku- and Ka-bands with independent feeds," IEEE Trans. Antennas Propag., vol. 65, no. 6, pp. 3241-3246, Jun. 2017.

[41] J. A. Encinar, R. Florencio, M. Arrebola, M. A. Salas, M. Barba, R. R. Boix, and G. Toso, "Dual-polarization reflectarray in Ku-band based on two layers of dipole-arrays for a transmit-receive satellite antenna with south american coverage," in $11^{\text {th }}$ European Conference on Antennas and Propagation (EuCAP), Paris, France, Mar. 19-24, 2017, pp. 80-83.

[42] R. Florencio, R. R. Boix, E. Carrasco, J. A. Encinar, M. Barba, and G. Pérez-Palomino, "Broadband reflectarrays made of cells with three coplanar parallel dipoles," Microw. Opt. Technol. Lett., vol. 56, no. 3, pp. 748-753, Mar. 2014.

[43] R. Florencio, J. A. Encinar, R. R. Boix, G. Pérez-Palomino, and G. Toso, "Cross-polar reduction in reflectarray antennas by means of element rotation," in $10^{\text {th }}$ European Conference on Antennas and Propagation (EuCAP), Davos, Switzerland, Apr. 10-15, 2016, pp. 1-5.

[44] R. Florencio, R. R. Boix, E. Carrasco, J. A. Encinar, and V. Losada, "Efficient numerical tool for the analysis and design of reflectarrays based on cells with three parallel dipoles," Microw. Opt. Technol. Lett. vol. 55, no. 6, pp. 1212-1216, Jun. 2013.

[45] J. A. Nelder and R. Mead, "A simplex method for function minimization,” Comput. J., vol. 7, no. 4, pp. 308-313, Jan. 1965.

[46] J. C. Lagarias, J. A. Reeds, M. H. Wright, and P. E. Wright, "Convergence properties of the Nelder-Mead simplex method in low dimensions," SIAM J. Optim., vol. 9, no. 1, pp. 112-147, 1998.

[47] Y.-T. Lo and S.-W. Lee, Eds., Antenna Handbook Vol. 1. New York, NY, USA: Van Nostrand Reinhold, 1993, ch. 1, pp. 28-29.

[48] J. A. Encinar and M. Arrebola, "Reduction of cross-polarization in contoured beam reflectarrays using a three-layer configuration," in IEEE Antennas and Propagation Society International Symposium, Honolulu, Hawaii, USA, Jun. 9-15, 2007, pp. 5303-5306.

[49] C. Tienda, J. A. Encinar, M. Arrebola, M. Barba, and E. Carrasco, "Design, manufacturing and test of a dual-reflectarray antenna with improved bandwidth and reduced cross-polarization," IEEE Trans. Antennas Propag., vol. 61, no. 3, pp. 1180-1190, Mar. 2013.

[50] D. R. Prado, A. Campa, M. Arrebola, M. R. Pino, J. A. Encinar, and F. Las-Heras, "Design, manufacture and measurement of a low-cost reflectarray for global Earth coverage," IEEE Antennas Wireless Propag. Lett., vol. 15, pp. 1418-1421, 2016.

[51] M. Arrebola, J. A. Encinar, and M. Barba, "Multifed printed reflectarray with three simultaneous shaped beams for LMDS central station antenna," IEEE Trans. Antennas Propag., vol. 56, no. 6, pp. 1518-1527, Jun. 2008.

[52] M. Sato, "OpenMP: parallel programming API for shared memory multiprocessors and on-chip multiprocessors," in $15^{\text {th }}$ International Symposium on System Synthesis, Kyoto, Japan, Oct. 2-4, 2002, pp. 109111.

[53] D. R. Prado, J. Álvarez, M. Arrebola, M. R. Pino, R. G. Ayestarán, and F. Las-Heras, "Efficient, accurate and scalable reflectarray phase-only synthesis based on the Levenberg-Marquardt algorithm," Appl. Comp. Electro. Society Journal, vol. 30, no. 12, pp. 1246-1255, Dec. 2015.

[54] D. R. Prado, M. Arrebola, M. R. Pino, F. Las-Heras, R. Florencio, R. R. Boix, and J. A. Encinar, "Reflectarray antenna with reduced crosspolar radiation pattern," in $10^{\text {th }}$ European Conference on Antennas and Propagation (EuCAP), Davos, Switzerland, Apr. 10-15, 2016, pp. $1-5$. 


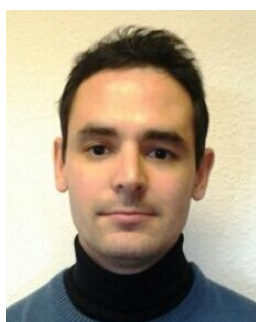

Daniel R. Prado was born in Sama de Langreo, Asturias, Spain, in 1986. He received the B.Sc., M.Sc., and Ph.D. degrees in telecommunication engineering from the University of Oviedo, Gijón, Spain in 2011, 2012, and 2016 respectively, where he is currently a Postdoc Researcher.

Since 2011, he has been working as a Research Assistant with the Signal Theory and Communications Area, University of Oviedo. From September 2010 to March 2011 he was with The Institute of Electronics, Communications and Information Technology (ECIT), Queen's University Belfast, UK, working on the design of leaky-wave antennas as part of his B.Sc. research project. From August to November 2014 he was with the School of Electrical Engineering, KTH Royal Institute of Technology, Stockholm, Sweden, as a Visiting Scholar, where he worked in transformation optics applied to dielectric lenses. His current research activities include analysis of non-uniform arrays, and the development of efficient techniques for the analysis and optimization of near and far fields of reflectarray antennas.

Dr. Prado was the recipient of a scholarship financed by Gobierno del Principado de Asturias.

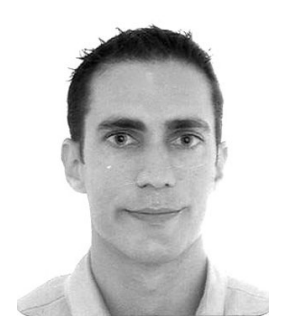

Jesús A. López-Fernández was born in Avilés, Asturias, Spain. He received the M.Sc. and the Ph.D. degrees in telecommunication engineering from the University of Vigo, Spain, in 1999 and 2009, respectively.

From April 2002 to March 2003, he was a MarieCurie Visiting Fellow at the Mechanical \& Manufacturing Engineering Department, Trinity College Dublin (TCD). Since October 2003, he has been with the Electrical Engineering Department, University of Oviedo, Asturias, Spain, where he is currently an Associate Professor teaching courses on Digital Communications and Radar Systems. His research interests include iterative methods and speedup schemes applied to scattering problems, parallel algorithms, and signal processing techniques.

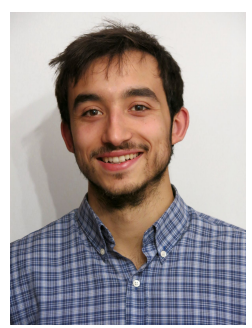

Guillermo Barquero was born in Mieres, Asturias, Spain in 1992. He received the B.Sc. and M.Sc. degrees in telecommunication engineering from the University of Oviedo, Gijón, Spain, in 2014 and 2016, respectively.

In 2015 he was with the Signal Theory and Communications Area, University of Oviedo, working on machine learning applied to antenna design. Since 2016, he has been working as a Data Scientist in ArcelorMittal Global R\&D in Avilés, Spain. His research interests include Multivariate Time Series analysis and the industrialization of traditional machine learning algorithms with a special focus on bringing model interpretability along with accurate results.

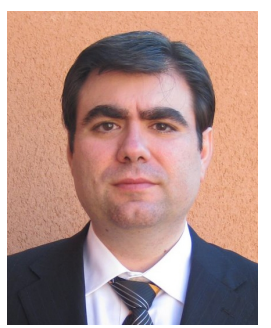

Manuel Arrebola (S'99-M'07-SM'17) was born in Lucena (Córdoba), Spain. He received the Ingeniero de Telecomunicación degree from the Universidad de Málaga (UMA), Málaga, Spain, in 2002, and the $\mathrm{Ph} . \mathrm{D}$. degree from the Universidad Politécnica de Madrid (UPM), Madrid, Spain, in 2008.

From 2003 to 2007, he was with the Electromagnetism and Circuit Theory Department at UPM as a Research Assistant. From August to December 2005, he was with the Microwave Techniques Department at the Universität Ulm, Ulm, Germany, as a Visiting Scholar. In December 2007, he joined the Electrical Engineering Department at the Universidad de Oviedo, Gijón, Spain, where he is an Associate Professor. In 2009, he enjoyed a two-month stay at the European Space Research and Technology Centre, European Space Agency (ESTEC-ESA), Noordwijk, The Netherlands. His current research interests include analysis and design techniques of contoured-beam and reconfigurable printed reflectarrays both in single and dual-reflector configurations and planar antennas.

Dr. Arrebola was co-recipient of the 2007 S.A. Schelkunoff Transactions Prize Paper Award, given by the IEEE Antennas and Propagation Society.

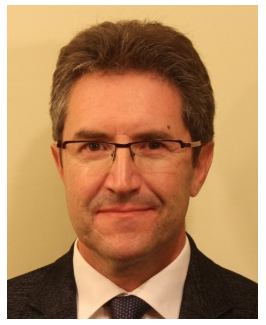

Fernando Las-Heras (M'86-SM'08) received the M.Sc. degree in 1987 and the Ph.D. degree in 1990, both in telecommunication engineering, from the Technical University of Madrid, Spain (UPM).

From 1988 to 1990 , he was a National Graduate Research Fellow. From 1991 to 2000 he held a position of Associate Professor at the Department of Signal, Systems and Radiocommunications, UPM. Since December 2003, he holds a Full-Professor position at the University of Oviedo where he was the Vice-Dean for Telecommunication Engineering at the Technical School of Engineering in Gijón from 2004 to 2008. As of 2001, he heads the research group Signal Theory and Communications TSCUNIOVI, Department of Electrical Engineering, University of Oviedo. He was a Visiting Lecturer at the National University of Engineering in Peru in 1996, a Visiting Researcher at Syracuse University, New York, USA, in 2000, and a short term Visiting Lecturer at ESIGELEC in France from 2005 to 2011. From 2005, he holds the Telefónica Chair ICTs Applied to Environment and Climate Change at the University of Oviedo and from 2010 he is a member of the Science, Technology and Innovation Council of Asturias, and member of the board of directors of the IEEE Spain Section. He has authored over 300 articles published in academic journals and proceedings of international conferences, mainly in the areas of antenna design and the inverse electromagnetic problem with applications in diagnostic, measurement and synthesis of antennas, phaseless techniques, propagation, and microwave to $\mathrm{THz}$ imaging and localization, as well as in engineering education. 